\title{
RECENT DEVELOPMENTS IN THE THEORY OF WALSH SERIES
}

\author{
WILLIAM R. WADE \\ Department of Mathematics \\ University of Tennessee \\ Knoxville, Tennessee 37916 U.S.A. \\ (Received May 20, 1982)
}

ABSTRACT. We survey research done on the theory of Walsh series during the decade 1971-1981. Particular attention is given to convergence of Walsh-Fourier series, gap walsh series, growth of Walsh-Fourier coefficients, dyadic differentiation, and uniqueness of Walsh series.

KEY WORDS AND PHRASES. Walsh-Paley system, dyadic group, maximal functions, dyadic $H^{p}$ spaces, BMO, Vilenkin groups, weak type $(p, p)$, dyadic differentiation. 1980 MATHEMATICS SUBJECT CLASSIFICATION CODES. 42C10, $43 A 75$.

I. INTRODUCTION.

This article surveys recent results on Walsh series. To avoid duplication of material appearing in Balašov and Rubinštein [1970], a decision was made to concentrate on the decade 1971-1981. References to earlier work will be made when necessary to relate what is herein reported to that which preceeded it. Discussion of the relationships between this material and the general theory of orthogonal series has been left to those more qualified for this task (e.g., UI'janov [1972], 0levski $\breve{i}$ [1975] and Bočkarev [1978b]).

In addition to this introductory section, there remain five sections: II. Walsh-Fourier Series, III. Approximation by Walsh Series, IV. Walsh-Fourier Coefficients, V. Dyadic Differentiation, and V. Uniqueness. These sections have been further divided into consecutively numbered subsections, each dealing with a 
particular facet of the subject and each carrying a descriptive title to help the reader quickly find those parts which interest him most.

Section VI is followed by a nearly complete listing of all articles on Walsh series published during the decade 1971-1981. This listing is ordered alphabetically and then chronologically. In the course of our narrative these articles will be cited, as above, by author and by year.

Let $r_{0}, r_{1}, \ldots$ represent the Rademacher functions, i.e.,

$$
r_{k}(x)=\operatorname{sgn}\left(\sin \left(2^{k+1} \pi x\right)\right), k \geq 0, x \in[0,1] .
$$

Let $w_{0}, w_{1}, \ldots$ represent the walsh functions, i.e., $w_{0} \equiv 1$ and if $k=2^{n_{1}}+\ldots+2^{n_{\gamma}}$ is a positive integer with $n_{1}>n_{2}>\ldots>n_{\gamma} \geq 0$ then

$$
w_{k}(x)=r_{n_{1}}(x) \ldots r_{n_{\gamma}}(x) \text {. }
$$

The idea of using products of Rademacher functions to define the Walsh system originated with Paley [1932]. Thus the system $\left\{w_{k}\right\}$ as defined above is called the WalshPaley system. It is interesting to note that this process of putting factors together to make an orthonormal system only produces "Walsh-like" functions. Indeed, Waterman $[969],[1982]$ proved that if $\phi_{0}, \phi_{1}, \ldots$ is a system of real functions on $[0,1]$ which satisfies $\left|\phi_{n}\right| \leq 1$ a.e., $n \geq 0$, if $\psi_{k}=\phi_{n_{1}} \ldots \phi_{n_{\gamma}}$ for $k=2^{n_{1}}+\ldots+2^{n_{\gamma}}$ defines an orthogonal system with $\left|\psi_{k}\right|=1$ a.e., and if $\int \psi_{k}{ }^{2} \equiv M$ for $k$ sufficiently large, then there exists a measure preserving map $\lambda$ of $[0,1]$ onto itself such that $w_{k} \circ \lambda=\psi_{k}$ a.e. for $k=1,2, \ldots$ Moreover, the system $\left\{\psi_{k}\right\}$ is complete if and only if the map $\lambda$ can be chosen to be a metric automorphism of $[0,1]$.

By a Walsh series we shall mean a series of type $W=\sum_{k=0}^{\infty} a_{k} w_{k}$, where $a_{0}, a_{1}, \ldots$ are real numbers. The $\underline{n}^{\text {th }}$ partial sums of $W$ will be denoted by

$$
w_{n}=\sum_{k=0}^{n-1} a_{k} w_{k}, n \geq 1
$$

By a Walsh-Fourier series we shall mean a Walsh series of the form $W[f]=\sum_{k=0}^{\infty} a_{k}(f) w_{k}$ where $a_{0}(f), a_{1}(f), \ldots$ represent the Walsh-Fourier coefficients of some integrable f, i.e., 


$$
a_{k}(f)=\int_{0}^{1} f(t) w_{k}(t) d t, k \geq 0 .
$$

Let $L^{p}, 1 \leq p<\infty$, represent the collection of measurable functions whose $p^{\text {th }}$ power is integrable over the interval $[0,1]$. Let $L^{\infty}$ represent the collection of measurable functions whose essential supremum is finite on the interval $[0,1]$, and let $\mathscr{E}$ represent the collection of functions continuous on the interval $[0,1]$. It is well known that the Walsh functions form a complete orthonormal system in $L^{2}$.

It is clear that the Walsh functions al ternate between +1 and -1 on the interval $[0,1]$ and it is not difficult to see that the $2^{n}$ th partial sums of the Dirichlet kernel $D=\sum_{k=0}^{\infty} W_{k}$ are always non-negative. These properties penetrate deeply into the very essence of the Walsh system. Indeed, Levizov [1980] has shown that any orthonormal system whose functions $f_{n}$ have exactly $n$ sign changes on $[0,1]$, have range $\{+1,-1\}$, and satisfy $f_{n}(0)=0$, for $n=0,1, \ldots$, is the Walsh system. And, Price [1961] proved that among the orthonormal systems whose functions $f_{n}$ alternate signs on finer and finer partitions of $[0,1]$, as $n \rightarrow \infty$, the Walsh system is the only one whose Dirichlet kernel has non-negative $2^{n}$ th partial sums. Thus these two features distinguish the study of Walsh series from that of other orthonormal systems.

Another distinguishing feature is that the Walsh functions can be identified with the characters of a certain compact group, $2^{\omega}$. This group, called the dyadic group, is the cartesian product of countably many copies of the discrete group $\{0,1\}$ endowed with the product topology. Thus a typical element of the dyadic group is a sequence $\left(x_{1}, x_{2}, \ldots\right)$ with each $x_{j}=0$ or $1, j \geq 1$. The map

$$
\lambda\left(x_{1}, x_{2} \ldots\right)=\sum_{j=1}^{\infty} x_{j} 2^{-j}
$$

identifies the dyadic group with the interval $[0,1]$ much like the map $e^{i x} \rightarrow x$ identifies the circle group $T$ with the interval $[0,2 \pi]$. It turns out that if $\Psi_{0}, \Psi_{1}, \ldots$ represent the characters of the dyadic group, then $\Psi_{k}=w_{k} \circ \lambda$ for $k=0,1, \ldots$. Thus each $w_{k} \circ \lambda$ is continuous on the group and satisfies $w_{k} \circ \lambda(\bar{x}) w_{k} \circ \lambda(\bar{y})=w_{k} \circ \lambda(\bar{x}+\bar{y})$ for $\bar{x}, \bar{y} \in 2^{\omega}$. This allows one to use theorems about Fourier analysis on the dyadic group to solve problems about Walsh-Fourier analys is on the interval $[0,1]$. It also allows one to pull the dyadic group structure back to the interval $[0,1]$, defining a 
dyadic sum of two numbers $x, y, \in[0,1]$ by

$$
x+y=\lambda(\bar{x}+\bar{y})=\sum_{k=1}^{\infty}\left|x_{k}-y_{k}\right| 2^{-k},
$$

where $\bar{x}=\left(x_{1}, x_{2}, \ldots\right)$ and $\bar{y}=\left(y_{1}, y_{2}, \ldots\right)$ come from the binary expansion of $x$ and $y$ and the finite expansion is used when $x$ or $y$ is a dyadic rational. The character property of $w_{k}$ oג means that $w_{k}(x+y)=w_{k}(x) w_{k}(y)$ holds for $x, y_{1} \in[0,1], k \geq 0$. In particular, one can almost view $([0,1], \dot{+})$ as a compact group whose characters are $w_{0}, w_{1}, \ldots$ and do most Walsh analysis there. The word "almost" is necessary because $\lambda$ is not quite $1-1$. Indeed $\lambda^{-1}(x)$ has two distinct images for each dyadic rational $x$; one corresponds to the finite expansion and one corresponds to the infinite expansion, e.g. $\lambda(1,0,0, \ldots)=\lambda(0,1,1, \ldots)=1 / 2$. Thus $[0,1]$ is not a compact group under $\dot{+}$ unless each dyadic rational is split into two points. (This point of view was introduced by Šneider [1949].)

Let $f$ be defined on $[0,1]$. We shall represent the dyadic moduli of continuity by

$$
\dot{\omega}(\delta, f)=\sup _{\substack{0<h<\delta \\ x \in[0,1]}}|f(x+h)-f(x)|
$$

and

$$
\dot{\omega}_{p}(\delta, f)=\sup _{0 \leq h<\delta}\left\{\int_{0}^{l}|f(x+h)-f(\dot{x})|^{p} d x\right\}^{1 / p},
$$

$1 \leq p<\infty, \delta>0$. The function $f$ belongs to $\operatorname{Lip}\left(\alpha, L^{p}\right)$ for some $0<\alpha \leq 1$ if there exists a constant $C$ such that $\dot{\omega}_{p}(\delta, f) \leq C \delta^{\alpha}$ for $\delta>0$. Similarly $f$ belongs to Lip $\alpha$ if $|f(x+h)-f(x)| \leq C h^{\alpha}$ for $h \in[0,1]$.

A most useful distinguishing feature of the Walsh system is that the $2^{n}$ th partial sums of any walsh series form a martingale. In particular, theorems about martingales contain information about $2^{n}$ th partial sums of Walsh series. Thus, given any $f \in L^{1}$, its (martingale) maximal function is

$$
f^{\star}(x)=\sup _{n>0}\left|w_{2} n[f, x]\right| x \in[0,1]
$$

its square function is

$$
S(f)=\sqrt{\sum_{k=1}^{\infty}\left(w_{2} k[f]-W_{2} k-1[f]\right)^{2}},
$$


and it is well known (see Garsia [1973]) that $\left\|f^{\star}\right\|_{L} \leq \sqrt{10}\|S(f)\|_{L}$ and $\|S(f)\|_{L^{1}} \leq 5\|f *\|_{L^{1}}$. The function $f$ is said to belong to dyadic $H^{1}$ if

$$
\|f\|_{H^{1}} \equiv\|S(f)\|_{L^{1}}
$$

is finite. Thus $f$ belongs to dyadic $H^{\top}$ if and only if $f^{\star}$ is integrable.

Analogous to the classical case, the dual dyadic $H^{7}$ is the space of functions of dyadic bounded mean oscillation, dyadic BMO. This is the space of functions $f \in L^{1}$ for which the norm

$$
\|f\|_{B M O} \equiv \sup \left\{\frac{1}{m(I)} \int_{I}\left|f(x)-f_{I}\right| d x: \text { I is a dyadic interva }\right\}
$$

is finite. Included in this duality is the "Hölder" inequality of Fefferman:

$$
\left|\int_{0}^{1} f(x) \Phi(x) d x\right| \leq \sqrt{2}\|f\|_{H}{ }^{1}\|\Phi\|_{B M O} .
$$

This inequality only holds if the integral above is interpreted as

$$
\int_{0}^{1} f(x) \Phi(x) d x=\lim _{n \rightarrow \infty} \int_{0}^{1} W_{2} n[f, x] W_{2} n[\Phi, x] d x .
$$

Details and references can be found in Garsia [1973]. As in the classical case, dyadic $H^{1}$ can be characterized by atomic decompositions (see Chao [1982b]) and dyadic BMO enjoys the Carleson decomposition (see Chao [1982a]). Both of these decompositions provide easy proofs of the duality between dyadic $H^{l}$ and dyadic BMO.

The space dyadic $H^{1}$ is not only analogous to the classical Hardy space $H^{1}$, but nearly equivalent to it. Indeed, by the atomic theory of Coifman and Weiss [1977] it is clear that dyadic $H^{1}$ is a (proper) subset of classical $H^{1}$. On the other hand, a recent result of Davis [1980] proves that given $f$ in classical $H^{1}$, its translates ${ }^{\tau} \mathrm{f}$ belong to dyadic $H^{1}$ for a.e. $x$.

Vilenkin [1947] showed that Walsh series and the group $2^{\omega}$ are a special case of a broad theory of Fourier analys is on zero-dimensional groups. Indeed, he proved that if $G$ is any compact, abelian, zero-dimensional group which satisfies the second axiom of countability, then there exist primes $p_{1}, p_{2}, \ldots$ such that each $\bar{x} \in G$ can be identified with a sequence $\left(x_{1}, x_{2}, \ldots\right)$ of integers where $0 \leq x_{j}<p_{j}$. Thus the group $G$ can be identified with $[0,1]$. Indeed, for each integer $k \geq 1$ set $m_{k}=\pi_{j=1}^{k} p_{j}$. 
Given $\bar{x}=\left(x_{1}, x_{2}, \ldots\right)$ let $x=\sum_{j=1}^{\infty} x_{j} / m_{j}$. Then $x \in[0,1]$ and except for a countable set, the map $\bar{x} \rightarrow x$ is $1-1$ and takes $G$ onto $[0,1]$. Vilenkin also introduced a natural ordering for the characters $\Psi_{0}, \Psi_{1}, \ldots$ of $G$. In the case when all $p_{j}=2$, the group $G$ is precisely the dyadic group and the characters $\psi_{0}, \psi_{1}, \ldots$ are precisely $w_{k} \circ \lambda$, for $k=0,1, \ldots$.

We shall denote the Haar measure of a Vilenkin group $G$ by $m$. We shall denote character series $\sum_{k=0}^{\infty} a_{k} \Psi_{k}$ by $S$ and Fourier series on $G$ by $S[f]$. The context and the square brackets will keep this from being confused with the square function $S(f)$ defined above. We shall denote the $L^{p}$ spaces on $G$ with respect to $m$ by $L^{p}(G)$. The moduli of continuity on $G$ will be denoted by

and

$$
\omega_{k}(f)=\sup _{h \in G_{k}, x \in G}|f(x+h)-f(x)|
$$

$$
\omega_{k}^{(p)}(f)=\sup _{h \in G_{k}}\left\{\int_{G}|f(x+h)-f(x)|^{p d m}(x)\right\}^{1 / p},
$$

where $G_{k} \equiv\left\{\bar{x} \in G: x_{j}=0\right.$ for $\left.j<k\right\}, k=1,2, \ldots$. Using the identificaton of $G$ with $[0,1]$ we can also define G-moduli of continuity for functions $f$ with domain $[0,1]$. These will be denoted as above by $\omega(\delta, f)$ and $\omega_{p}(\delta, f)$. A vilenkin group $G$ is said to be of bounded type if $\limsup _{n \rightarrow \infty} p_{n}<\infty$. Although the proofs usually require greater sophistication and often necessitate the introduction of new techniques, the theory for Vilenkin groups of bounded type parallels that for Walsh series. This is not the case for Vilenkin groups of unbounded type. In fact very little is known about these groups.

An important example of Vilenkin groups is provided by the ring of integers $\mathscr{D}$ of any local field $K$. $\mathscr{D}$ is the maximal compact subring in $K$, and when $K$ is the 2-series field, the ring $y$ is precisely the group $2^{\omega}$. This point of view provides a vehicle for discussing dyadic analogues of Riesz transforms, Bessel potentials, Mellin and Hankel transforms, and certain singular integral operators. An elegant, clearly written introduction to harmonic analysis on local fields is provided by Taibleson [1975]. For $H^{P}$ spaces in this setting see Chao [1975] - [1982b] and Chao, Gilbert and Tomas [1981]. For an analogue of the F and M Riesz theorem, see Chao and Taibleson [1979]. During the course of our narrative we shall have occasion to cite certain re- 
sults about Fourier analys is on Vilenkin groups or local fields. In some cases the cited result will generalize a walsh series result discussed in that section. In other cases, the cited works will give new information about walsh series. In such cases, we leave it to the reader to extract this new information.

Finally, we do not systematically discuss the many applications of Walsh series to physical problems. We suggest Maqusi [1981] and Harmuth [1972] for general references. Concerning sampling theorems see Butzer and Splettstösser [1978], [1980], and Spletts tösser [1979].

\section{WALSH-FOURIER SERIES}

1. Pointwise convergence. Billard [1967] showed that the Walsh-Fourier series of $L^{2}$ functions converge a.e. His proof was a dyadic group adaptation of the CarlesonHunt technique which reduces the problem of a.e. convergence to showing that the maximal function operator $f \rightarrow M f \equiv \sup _{n>0}\left|W_{n}[f]\right|$ is of type $(2,2)$, i.e., that there exists a constant $A$ such that $\|M f\|_{2} \leq A\|f\|_{2}$ holds for all $f \in L^{2}[0,1]$. An excellent exposition of this proof has been provided by Hunt [1970]. A different approach to a.e. convergence of Walsh-Fourier series has been given by Gosselin [1979]. He avoids the tedious estimates necessary to show that Mf is of type $(2,2)$ by using Fefferman's $L^{2}$ method of breaking the partial sum operator into simpler pieces.

The best positive result of a.e. convergence of Walsh-Fourier series (and Fourier series as well) belongs to Sjölin [1969]. He derived certain weak type inequalities involving Mf to show that W[f] converges a.e. when $f \in \operatorname{Llog}^{+} \operatorname{Llog}^{+} \log ^{+} L$, i.e., when

$$
\int_{0}^{1}|f(x)| \log ^{+}|f(x)| \log ^{+} \log ^{+}|f(x)| d x<\infty .
$$

It follows that the Walsh-Fourier series of any function in $L\left(\log ^{+} L\right)^{1+\epsilon}, \epsilon>0$, converges a.e. It is an open question whether $W[f]$ is a.e. convergent for all $f \in \operatorname{Llog}^{+} L$.

In the negative direction, Ladhawala and Pankratz [1976] showed that there exists an $f$ in dyadic $H^{1}$ whose Walsh-Fourier series diverges a.e. Since a non-negative function belongs to dyadic $H^{1}$ if and only if it belongs to $L \log ^{+} L$, a modification of their example could provide a negative answer to the question cited in the previous paragraph. For the larger spaces $L\left(\log ^{+} \log ^{+} L\right)^{1-\epsilon}, \epsilon>0$, Moon [1975] has shown that Walsh-Fourier series can diverge everywhere. It is not yet known whether Walsh-Four- 
sults about Fourier analys is on Vilenkin groups or local fields. In some cases the cited result will generalize a walsh series result discussed in that section. In other cases, the cited works will give new information about walsh series. In such cases, we leave it to the reader to extract this new information.

Finally, we do not systematically discuss the many applications of Walsh series to physical problems. We suggest Maqusi [1981] and Harmuth [1972] for general references. Concerning sampling theorems see Butzer and Splettstösser [1978], [1980], and Spletts tösser [1979].

\section{WALSH-FOURIER SERIES}

1. Pointwise convergence. Billard [1967] showed that the Walsh-Fourier series of $L^{2}$ functions converge a.e. His proof was a dyadic group adaptation of the CarlesonHunt technique which reduces the problem of a.e. convergence to showing that the maximal function operator $f \rightarrow M f \equiv \sup _{n>0}\left|W_{n}[f]\right|$ is of type $(2,2)$, i.e., that there exists a constant $A$ such that $\|M f\|_{2} \leq A\|f\|_{2}$ holds for all $f \in L^{2}[0,1]$. An excellent exposition of this proof has been provided by Hunt [1970]. A different approach to a.e. convergence of Walsh-Fourier series has been given by Gosselin [1979]. He avoids the tedious estimates necessary to show that Mf is of type $(2,2)$ by using Fefferman's $L^{2}$ method of breaking the partial sum operator into simpler pieces.

The best positive result of a.e. convergence of Walsh-Fourier series (and Fourier series as well) belongs to Sjölin [1969]. He derived certain weak type inequalities involving Mf to show that W[f] converges a.e. when $f \in \operatorname{Llog}^{+} \operatorname{Llog}^{+} \log ^{+} L$, i.e., when

$$
\int_{0}^{1}|f(x)| \log ^{+}|f(x)| \log ^{+} \log ^{+}|f(x)| d x<\infty .
$$

It follows that the Walsh-Fourier series of any function in $L\left(\log ^{+} L\right)^{1+\epsilon}, \epsilon>0$, converges a.e. It is an open question whether $W[f]$ is a.e. convergent for all $f \in \operatorname{Llog}^{+} L$.

In the negative direction, Ladhawala and Pankratz [1976] showed that there exists an $f$ in dyadic $H^{1}$ whose Walsh-Fourier series diverges a.e. Since a non-negative function belongs to dyadic $H^{1}$ if and only if it belongs to $L \log ^{+} L$, a modification of their example could provide a negative answer to the question cited in the previous paragraph. For the larger spaces $L\left(\log ^{+} \log ^{+} L\right)^{1-\epsilon}, \epsilon>0$, Moon [1975] has shown that Walsh-Fourier series can diverge everywhere. It is not yet known whether Walsh-Four- 
[1975] showed that if $\omega$ is a non-negative function on $G$ which satisfies the $A_{p}$ condition, $p>1$, i.e., if

$$
\left(\frac{1}{m(E)} \int_{E} \omega d m\right)\left(\frac{1}{m(E)} \int_{E} \omega^{\frac{-1}{(p-1)}} d m\right)^{p-1} \leq B<\infty
$$

holds for each coset $E$ of the subgroups $G_{n}=\left\{\bar{x} \in G: x_{j}=0\right.$ for $\left.j<n\right\}, n \geq 0$, then there exists a constant $C_{p}<\infty$ such that

$$
\int_{G}|M f|^{p}{ }_{\omega d m} \leq C_{p} \int_{G}|f| \omega d m
$$

holds for all measurable $f$ on $G$. It follows that $S[f]$ converges a.e. when the right hand side of this inequality is finite.

For the case when $G$ is the dyadic group, Gundy and Wheeden [1974] proved that under the $A_{p}$ condition, $1<p<\infty$, the weighted $L^{p}$ norms of $f$ and of $i$ ts square function $S(f)$ are equivalent. Thus they extended the pioneering work of Hirschman [1955] who considered weights of the form $\omega(x)=|x|^{\alpha}$, for $-1<\alpha<p-1$. Gundy and Wheeden also considered the limiting case when $p=1$. They showed that if $\omega \geq 0$ satisfies the $A_{\infty}$ condition and if

$$
m_{\omega}(E) \equiv \int_{E} \omega d m
$$

then given $\alpha>1$ and $\beta>1$ there exists a number $\gamma$ such that

$$
\alpha \mathrm{m}_{\omega}\left(f^{\star}>\beta \lambda, S(f) \leq \gamma \lambda\right) \leq m_{\omega}\left(f^{\star}>\lambda\right), \lambda>0,
$$

and such that a similar inequality holds with the roles of $f^{*}$ and $S(f)$ reversed.

Concerning rearrangements of multiple walsh-Fourier series, Kemhadze [1975] proved that given $f \in L^{P}(G), p>1$, the multiple Walsh-Fourier series of $f$ can be rearranged so that its spherical partial sums converge to $f$ a.e.

2. Sets of divergence. Lukašenko [1978] studied proper sets of divergence, i.e., sets of the form

$$
E=\{x: W[f, x] \text { diverges }\}
$$

for some integrable $f$. He proved that given any $\mathscr{G}_{\delta}$ set $E$ there is a function $f \in L^{l}$ such that (3) holds. However, there are $\xi_{\delta}$ sets and $F_{\sigma}$ sets for which (3) never holds when $f \in \mathscr{C}$. Moreover, in [1980] he announced that there exists a continuous $f$ such that the set E defined by (3) is of Haar measure zero, is a $\mathscr{G}_{\delta \sigma}$ set, but is neither a $\mathscr{G}_{\delta}$ nor an $\mathscr{F}_{\sigma}$ set.

A set $E \subseteq[0,1]$ is said to be a set of divergence for a class $\mathscr{F}$ of integrable 
functions if there exists an $f \in \mathcal{F}$ such that $W[f]$ diverges on $E$. According to Sjölin's result (see $\S 1$ ), all sets of divergence for $L^{p}, p>1$, are of measure zero. This result is best possible since any set of measure zero is a set of divergence for $L^{p}, 1 \leq p<\infty$. Indeed, for Vilenkin groups of bounded type, Heladze [1978] has shown that every set $E \subseteq G$ of Haar measure zero is a set of divergence for $L^{p}(G)$, $1 \leq \mathrm{p}<\infty$. This problem is still open for $\mathrm{p}=\infty$ and for $\mathscr{C}(G)$. An $n$-dimensional version of Heladze's theorem was obtained by Sanadze and Heladze [1977].

For the trigonometric case, Kahane and Katznelson proved that any set of measure zero is a set of divergence for $\mathscr{C}$. This question remains open for Walsh series. Using the Banach-Steinhaus theorem, it is not difficult to prove that any countable set is a set of divergence for $\mathscr{E}\left(2^{\omega}\right)$. The first uncountable sets of divergence for $\mathscr{C}\left(2^{\omega}\right)$ were identified by Simon [1973]. His work is valid on any Vilenkin group $G$, and his sets of divergence have uncountable intersection with each subgroup $G_{n}$. Onneweer [1979a] proved that every set $E$ of logarithmic Hausdorff measure zero is a set of (bounded) divergence for $\mathscr{E}\left(2^{\omega}\right)$. Harris and the author [1978] have constructed certain perfect (uncountable) sets of divergence for $\mathscr{C}\left(2^{\omega}\right)$ but it is not yet known whether every perfect set of measure zero is a set of divergence for $\mathscr{C}\left(2^{\omega}\right)$.

3. Pointwise convergence of rearranged Walsh-Fourier series. First, we point out that although the Walsh-Fourier series of any $L^{2}$ function converges a.e., it is not necessarily a.e. convergent when rearranged. For example, Heladze [1978] proved that for every Vilenkin group $G$ of bounded type there exists an $f \in L^{2}(G)$ such that some rearrangement of $S[f]$ diverges unboundedly everywhere on $G$.

It is natural to ask whether a hypothesis stronger than "f $\epsilon L^{2}$ " will guarantee a.e. convergence of rearrangements of $W[f]$. For example, does there exist a monotone increasing sequence $\{\omega(k)\}$ of positive numbers such that the condition $\left[a_{k}{ }^{2} \omega(k)<\infty\right.$ implies that a given walsh series $\left[a_{k} w_{k}\right.$ is a.e. unconditionally convergent? The answer is yes if $\omega(k)$ increases fast enough, e.g., $\omega(k)=k^{\alpha}$ for $\alpha>1$, because $\left(\sum\left|a_{k}\right|\right)^{2} \leq\left(\left[k^{-\alpha}\right)\left(\sum k^{\alpha} a_{k}{ }^{2}\right)\right.$. Tandori [1966] was first to obtain negative answers to this question: no if $\omega(k)=o(\operatorname{loglogk})$. Nakata [1972], [1974], [1979] and Bočkarev [1978a] have obtained increasingly stronger negative answers. In particular, Nakata [1979] proved that given $\omega(k)$ which satisfies $\left[\left(1 /\left(k_{\omega}(k)\right)\right)<\infty\right.$, there exists a se- 
quence of real numbers $\left\{a_{k}\right\}$ such that $\left[a_{k}{ }^{2} \omega(k)<\infty\right.$ and such that the walsh series $\sum a_{k} w_{k}$ has an a.e. divergent rearrangement. Thus it is difficult to find conditions which guarantee that all rearrangements of a given Walsh series converge a.e.

However, certain rearrangements are more important than others. When dealing with Walsh series, the Kaczmarcz rearrangement is an important rearrangement, for historic reasons and for applications (see Bală̌ov and Rubiň̆tein [1970], and Harmuth [1972]). It belongs to the general class of dyadic block rearrangements, i.e., rearrangements $\tilde{w}_{0}, \tilde{w}_{1}, \ldots$ which satisfy

$$
\left\{\tilde{w}_{k}: 2^{n} \leq k<2^{n+1}\right\}=\left\{w_{k}: 2^{n} \leq k<2^{n+1}\right\}
$$

for $n=0,1, \ldots$

The Dirichlet kernel $\tilde{D}_{n}=\sum_{k=0}^{n-1} \tilde{w}_{k}$ in the Walsh-Kaczmarcz sys tem behaves worse than the Dirichlet kernel in the Walsh (-Paley) system in the sense that

$$
\limsup _{n \rightarrow \infty} \frac{\tilde{D}_{n}(x)}{\log n} \geq C>0 .
$$

Thus it is harder to prove a Walsh-Kaczmarcz-Fourier series converges, but easier to find divergent Walsh-Kaczmarcz-Fourier series.

Balašov [1971] has shown that if $\omega(n) \downarrow 0$, as $n \rightarrow \infty$, there exists an $f \in L^{1}$ such that

$$
\limsup _{n \rightarrow \infty} \frac{\left|\tilde{W}_{n}[f, x]\right|}{\omega(n) \log n}=+\infty \text { a.e., }
$$

where $\tilde{W}_{n}[f]$ represents the $n$th partial sum of the Walsh-Kaczmarcz-Fourier series of f. In particular $\tilde{W}^{\tilde{f}}[f]$ can diverge a.e. when $f \in L\left(\log ^{+} L\right)^{1-\epsilon}$ for $\epsilon>0$. On the other hand, Young [1974b] proved that if $f \in L\left(\log ^{+} L\right)^{2}$ then $W[f]$ converges a.e. No one has yet improved either of these results. In particular, the problem of a.e. convergence of Walsh-Kaczmarcz-Fourier series remains open for $f \in L\left(\log ^{+} L\right)^{p}, 1 \leq p<2$.

Young [1974a] introduced a large class of dyadic block rearrangements and showed that $W[f]$ remains a.e. convergent under these rearrangements for $f \in L^{2}$. She also proved that a smaller class of dyadic block rearrangements preserves a.e. convergence of $W[f]$ when $f \in L\left(\log ^{+} L\right)^{2} \log ^{+} \log ^{+} L$. This program was carried out for Vilenkin groups of bounded type by Gosselin and Young [1975]. The techniques are CarlesonHunt and involve proving certain maximal inequalities of Hardy-Littlewood type for these rearrangements. 
An easier program for studying a.e. convergence of rearranged Walsh-Fourier series has been initiated by Schipp [1975]. If $n=\sum_{j=0}^{\infty} n_{j} 2^{j}$ and $m=\sum_{j=0}^{\infty} m_{j} 2^{j}$ are integers written in binary notation, denote the integer $\sum_{j=0}^{\infty}\left|n_{j}-m_{j}\right| 2^{j}$ by $n+m$. He calls a rearrangement $\left\{\tilde{w}_{k}\right\}$ of the walsh functions linear if there exists a permutation $T$ of integers such that $T(n+m)=T(n)+T(m)$ and $\tilde{w}_{k}=w_{T k}$ for $k \geq 0$. He shows directly that the partial sums of Walsh-Fourier series in linear rearrangements are closely related to partial sums in the usual (Paley) ordering. Thus he can apply Sjölin's estimates without repeating the lengthy Carleson-Hunt argument. In particular, he shows that linear rearrangements of $W[f]$ converge a.e. when $f \in L^{p}$, $p>1$. The Kaczmarcz rearrangement is only piecewise linear. Thus he modified his procedure to show that piecewise linear rearrangements of $\mathrm{W}[f]$ converge a.e. when $f \in L^{2}$. Bahšecjan [1979] has generalized this result from piecewise linear rearrangements to piecewise isomorphic rearrangements.

Skvorcov [1981b] proved a theorem about Walsh-Kaczmarcz-Fourier series which contains the following corollaries. If $f$ is continuous on the group and if $\dot{\omega}(\delta, f)=$ $o(1 / \log (1 / \delta))$ as $\delta \rightarrow 0$ then $W[f]$ is uniformly convergent. (Thus the Dini-Lipschitz test works for Walsh-Kaczmarcz series). If $f$ is continuous on the group and of bounded variation over $[0,1]$, then $\tilde{W}[f]$ is uniformly convergent. He also shows that localization does not hold for convergence or $(C, 1)$ summability of Walsh-Kaczmarcz series. Indeed, he constructed an $f \in L^{1}$ which equals 0 on $[0,1 / 2]$ but whose Walsh-Kaczmarcz-Fourier series is not $(C, 1)$ summable at the point $x=0$.

4. Convergence in norm. Paley [1932] proved that if $f \in L^{p}, 1<p<\infty$, then $W_{n}[f] \rightarrow f$ in $L^{P}$ norm, as $n \rightarrow \infty$. Watari [1958] obtained this same result for Vilenkin groups of bounded type. But it is now known to hold for any Vilenkin group. Indeed, Young [1976a], Simon [1976], and Schipp [1976d] all showed that if $G$ is any Vilenkin group and $f \in L^{p}(G)$ for $p>1$ then $S_{n}[f] \rightarrow f$ in $L^{p}$ norm, as $n \rightarrow \infty$. Of the three methods, Schipp's is most general and applies to certain arrangements of any product sys tem, but Young's is simplest.

These results extend neither to $p=\infty$ nor to $p=1$. Thus in order to conclude that $W_{n}[f] \rightarrow f$ in $L^{1}$ norm one needs an assumption in addition to $f \in L^{1}$. Onneweer [1978a] obtained an $L^{1}$ analogue of the Dini-Lipschitz test, for the Walsh-Paley 
ordering and Skvorcov [1981c] did the same for the Kaczmarcz ordering. Specifically, they showed if $\dot{\omega}_{1}(\delta, f)=o(1 / \log (1 / \delta))$, as $\delta \rightarrow 0$, then $W_{n}[f] \rightarrow f$ in $L^{1}$ norm, as $n \rightarrow \infty$. This test fails when " 0 " is replaced by "0". A Vilenkin group version of this test was obtained by Simon [1979].

Simon [1978a] has introduced an analogue $\tilde{M}$ of the Hardy-Littlewood maximal function (see Stein and Weiss [1971]) on Vilenkin groups $G$, showing that $\tilde{M}$ is of type $(p, p)$ for $1<p<\infty$ and of weak type $(1,1)$. An application of his inequalities is that $\left\|S_{n}[f]\right\|_{p} \leq A_{p}\|f\|_{p}$ holds for $f \in L^{P}(G), 1<p<\infty, n \geq 0$, where $A_{p}$ is an absolute constant depending only on $p$. He also introduced an analogue $T$ of the conjugate function [1976] and has shown [1978b] that there is an absolute constant C such that

$$
\mid\{x \in G: \tilde{M f}(x) \leq y \text { and }|T f(x)|>\lambda y\} \mid \leq C e^{-c \lambda y}
$$

holds for all $f \in L^{\prime}(G)$ and all $\lambda, y>0$.

Results concerning uniform convergence of Walsh-Fourier series can be found in the following two sections.

5. Moduli of continuity and absolute convergence of Walsh-Fourier series.

A classical result of Fine [1949] is that if $f \in L i p \alpha, \alpha>1 / 2$, then $W[f]$ converges absolutely. In this section we report several extensions and refinements of this result.

Yoneda [1973] weakened the condition " $f \in \operatorname{Lip} \alpha, \alpha>1 / 2$ " to convergence of the series

$$
\sum_{n=1}^{\infty} \dot{w}_{2}(1 / n, f) / \sqrt{n},
$$

and obtained the Walsh analogue of a theorem of Zygmund: if $f$ is continuous and of bounded variation and if

$$
\sum_{n=1}^{\infty} \sqrt{\dot{w}(1 / n, f) / n}<\infty
$$

then $W[f]$ is absolutely convergent. He also introduced a summability method $\tilde{A}$ and localized Fine's result in the following manner. If $f \in \operatorname{Lip} \alpha$ on some interval I, where $\alpha>1 / 2$, then $W[f]$ is absolutely summable $A$ on $I$.

In connection with (5), Bočkarev [1978b] has shown that if $\omega$ is any modulus of continuity which satisfies 


$$
\sum_{n=1}^{\infty} \sqrt{\omega(1 / n) / n}=+\infty
$$

then there exists an absolutely continuous $f$ with $\omega(\delta, f)=0(\omega(\delta))$, as $\delta \rightarrow 0$, such that $\left[\left|a_{k}(f)\right|=+\infty\right.$.

Onneweer [1972] generalized Fine's result to Vilenkin groups $G$ of bounded type by examining the role oscillation plays in determining absolute convergence of $S[f]$. Before stating his results we need some additional terminology. Let $f$ be defined on $G$ and let $H$ be a subset of $G$. The oscillation of $f$ on $H$ is defined by

$$
\operatorname{osc}(f, H)=\sup _{x_{1}, x_{2} \in H}\left|f\left(x_{1}\right)-f\left(x_{2}\right)\right| \text {. }
$$

For each integer $k>0$, let $z_{q, k}\left(0 \leq q<m_{k}\right)$ represent points of $G$ for which $z_{q}, k$ $+G_{k}$ exhaust the cosets of $G_{k}$ in $G$. The function $f$ is said to be of p-generalized bounded fluctuation if the terms of the series

$$
\sum_{k=1}^{\infty}\left(\left.\sum_{q=0}^{m_{k}-1} \operatorname{losc}\left(f, z_{q, k}+G_{k}\right)\right|^{p}\right)^{1 / p}
$$

are uniformly bounded in $k$. Onneweer proved that if $f \in L^{p}(G), 1 \leq p \leq 2$ and if (6) converges then $S[f]$ converges absolutely. He also showed that $S[f]$ is absolutely convergent when $f \in \operatorname{Lip} \alpha \alpha>0$, and is of $p$-generalized bounded fluctuation.

Onneweer [1974] proved that if $f$ belongs to $\operatorname{Lip}(\alpha, p) * \operatorname{Lip}(\beta, q)$ for $i \leq p \leq$ 2, $q>1,0<\alpha, \beta \leq 1$ and $(\alpha+\beta) p>1$ on some Vilenkin group $G$ of bounded type, then $S[f]$ converges absolutely. Quek and Yap [1981] showed that the hypothes is "of bounded type" is redundant. Their method rests on a powerful factorization of $\operatorname{Lip}(\alpha$, $r)$ as $\operatorname{Lip}(\alpha-\beta, r) * \operatorname{Lip}((\beta-1) / q, p)$ where $1 / p+1 / q=1$ and $q>1 / \beta$. It follows that Lip $(\alpha, r) \subseteq \operatorname{Lip}(\alpha+1 / s-1 / r, s)$ holds on any Vilenkin group when $0<\alpha<\infty$, $1 \leq r<s<\infty$ and $\alpha+1 / s>1 / r$.

Vilenkin and Rubinstein [1975] established Vilenkin group versions of results cited in the second paragraph of this section. Specifically, if $G$ is of bounded type then $S[f]$ is absolutely convergent when either

$$
\sum_{k=1}^{\infty} \sqrt{m_{k+1}} \omega_{k}^{(2)}(f)<\infty, f \in L^{2}(G)
$$

or when $f$ is continuous and of bounded variation on $G$ and

$$
\sum_{k=1}^{\infty} \sqrt{\omega_{k}(f)}<\infty
$$


holds. In particular, if $f \in L i p \alpha, \alpha>0$ and if $f$ is of bounded variation on $G$, then S[f] converges absolutely on $G$. (The reason that $(4),(7)$ and $(5),(8)$ are not exact analogues is that on the dyadic group, $\omega_{k}(f)$ corresponds to $\omega\left(2^{-k}, f\right)$ not $\omega(1 / k, f))$. McLaughlin [1973] has obtained exact Vilenkin group analogues of (4) and (5) (see §12.) All these results hold for Vilenkin groups of unbounded type as well (see Quek and Yap [1980], [1981]).

Combining results of Ladhawala [1976] and Butzer and Wagner [1973] one can show that $W[f]$ is absolutely convergent when $f \in \operatorname{Lip}\left(\alpha, L^{p}\right), \alpha>1, p>1$. It is an open question whether this is true for $\alpha=1$.

Finally, Rubinštein [1978] has shown that given any sequence $\omega_{n}+0$, as $n \rightarrow \infty$, and any Vilenkin group $G$ there exist functions $f_{1} \in L^{1}(G), f_{2} \in L^{2}(G)$, and $f_{\infty} \in \mathscr{C}(G)$ such that $\omega_{n}{ }^{(p)}\left(f_{p}\right)=\omega_{n}$ for $n \geq 0$ and $p=1,2$, or $\infty$.

6. Uniform convergence of Walsh-Fourier series. Onneweer [1970] obtained an analogue of a theorem of Salem. If $f$ is continuous and periodic of period 1 , and if the sequence

$$
\sum_{k=1}^{2-1} k^{-1}\left|f\left(x+\frac{2 k}{2^{n+1}}\right)-f\left(x+\frac{2 k+1}{2^{n+1}}\right)\right|
$$

converges to zero, as $n \rightarrow \infty$, then $W[f]$ converges uniformly on $[0,1]$. A similar result for Walsh-Kaczmarcz series is due to Skvorcov [1981b]. A version of this result also holds for Vilenkin groups $G$ of bounded type (Onneweer and Waterman [1971]). Thus, if $\omega_{k}(f)=o(1 / k)$, as $k \rightarrow \infty$, and if $f \in \mathscr{C}(G)$ then $S[f]$ is uniformly convergent on $G$ (Vilenkin [1947]).

For Vilenkin groups of bounded type, Onneweer and Waterman [1971] proved that if $f$ is continuous and of 1 -generalized bounded fluctuation (defined in $\$ 5$ above) then $S[f]$ is uniformly convergent. They strengthened this result in several directions in [1974]. In order to state their results we need additional terminology. Let $\Lambda=\left\{\lambda_{n}\right\}$ be a sequence of positive numbers which satisfy $\sum_{n=1}^{\infty} \lambda_{n}^{-1}=+\infty$. A function $f$ defined on $G$ is said to be of $\Lambda$-bounded fluctuation if there exists an $M<\infty$ such that for every collection $\left\{I_{n}\right\}$ of disjoint cosets in $G$ it is the case that

$$
\sum_{n=1}^{\infty} \lambda_{n}^{-1} \operatorname{osc}\left(f, I_{n}\right)<M \text {. }
$$


The function $f$ is said to be of harmonic bounded fluctuation if it is of $\{n\}$ - bounded fluctuation. Onneweer and Waterman showed that there exist functions $f$ of $\Lambda$-bounded fluctuation whose Vilenkin-Fourier series $S[f]$ diverge at at least one point. However, if $f$ is of harmonic bounded fluctuation then $S[f]$ converges at every point of continuity and converges uniformly on any closed set of continuity for $f$. Their method centered on finding an analogue of Lebesgue's test on $G$ and showing that this test is satisfied at points of continuity for functions of harmonic bounded fluctuation. Their work also contains a very interesting condition sufficient to conclude that a continuous function is of harmonic bounded fluctuation. Given an open set $V \subseteq G$, let $N(V)$ represent the number (possibly infinite) of disjoint open "intervals" separated by the elements of $G \sim V$. If $f \in \mathscr{E}(G)$ with range $[0,1]$ and if

$$
\int_{0}^{1} \log N\{x \in G: f(x)>y\} d y<\infty
$$

then $f$ is of harmonic bounded fluctuation. Thus such functions have uniformly convergent Vilenkin-Fourier series. This is a group analogue of the Garsia-Sawyer test (see Onneweer [1971b]).

Concerning the size of $\left|W_{n}[f]-f\right|$, Tevzadze [1978] announced certain esilinates in terms of the modulus of continuity and variation of $f$ which contain a group $2^{w}$ version of the Garsia-Sawyer test. Specifically, he states that if $v(n)$ represents the modulus of variation of $f$ then there is an absolute constant $C$ (independent of $f$ ) such that $\| f-\left.W_{n}[f]\right|_{L^{\infty}}$ is dominated by

$$
c \min _{1 \leq m \leq n}\left\{\omega(1 / n, f) \sum_{k=1}^{m} \frac{1}{k}+\sum_{k=m+1}^{n} \frac{v(k)}{k^{2}}\right\} .
$$

It follows that if $f$ belongs to the class $H^{\omega} \cap(v[v]$ for some modulus of continuity $w$ and some modulus of variation $v$, then $W[f]$ is uniformly convergent on $\left[0\right.$, : if $^{\text {if }}$ and only if the sequence (10) converges to zero, as $n \rightarrow \infty$. Hence for a given continuous $f \in V[v]$, a necessary and sufficient condition that $W[f]$ be uniformly convergent is that $\sum_{k=1}^{\infty} v(k) / k^{2}<\infty$.

Guličev [1980] (announced in [1979b]) has shown that the Walsh analogues of theorems of Oskolkov and Busko fail to hold. Specifically, if $\left\{L_{k}\right\}$ represent the Walsh-Lebesgue constants (See Fine [1949]) then 


$$
\liminf _{k \rightarrow \infty} \frac{\left\|f-W_{k}[f]\right\|_{\infty}}{\omega(1 / k, f) L_{k}}=0 \text { for } f \in \mathscr{C},
$$

and

$$
\liminf _{k \rightarrow \infty} \frac{\left\|W_{k}[f]\right\|_{\infty}}{L_{k}}=0 \text { for } f \in L^{\infty} .
$$

However, if the limit infima in (11) and (12) are taken over certain subsequences of integers then $=0$ is replaced by $>0$. Thus for certain subsequences, analogy with the trigonometric system is restored.

7. Summability of Walsh-Fourier series. Although there exist integrable functions whose Walsh-Fourier series diverge everywhere, certain averages of these WalshFourier series still approximate the given function.

For example, Fine [1955] showed that if $f \in L^{1}$ then $W[f]$ is a.e. $(C, \alpha)$ summable to $f$ for all $\alpha>0$. A new proof of this result has been given by Schipp [1976c]. He takes the Carleson-Hunt point of view, and shows that the maximal function associated with a large class of summability methods is of type $(\infty, \infty)$ and of weak type $(1,1)$. This same program was carried out for Vilenkin groups of bounded type by $P \hat{a} l$ and Simon [1977a]. In particular, if $\sigma_{n}(f)$ represents the $n^{\text {th }}$ partial Cesaro sum of $S[f]$ and if $\sigma^{*}(f)=\sup _{n>0}\left|\sigma_{n}(f)\right|$ then

$$
m\left\{x:\left|\sigma^{\star}(f, x)\right|>y\right\} \leq C\|f\|_{7} / y, \quad y>0 .
$$

Thus $f \in L^{l}(G)$ implies that $\sigma^{\star}(f)$ is weakly integrable. It is natural to ask under what conditions is $\sigma^{\star}(f)$ actually integrable? Nobuhiko [1979] used atomic $H^{\top}(G)$ to prove that

Hence $\sigma^{\star}(f) \in L^{\top}(G)$ when $f \in H^{1}(G)$.

$$
\left.\int_{G}\left|\sigma^{\star}(f)\right| d m \leq C\|f\|_{H}\right\rceil .
$$

Baiarstanova [1979] has considered $(C, 1)$ summability of $w_{n_{k}}[f]$ where $n_{k}=2^{k}+2^{k-2}+2^{k-4}+\ldots+1$. She showed that if $\dot{\omega}(\delta, f)$ (respectively, $\dot{\omega}_{1}(\delta, f)$ ) is $0(1 / \sqrt{\log (1 / \delta)})$, as $\delta \rightarrow 0$, then $w_{n_{k}}[f]$ is uniformly $(C, 1)$ summable (respectively, $(C, 1)$ summable in $L^{1}$ norm). This result contrasts nicely with Schipp's result (see (1) and (2) in \$1).

Other methods of averaging can also be used to show that $W[f]$ approximates $f$ in some sense. One such example is the method of strong summability. Schipp [1969a] proved that if $f \in L^{1}$ then 


$$
\frac{1}{m}\left\{\sum_{k=0}^{m-1}\left|w_{k}[f]-f\right|^{p_{j}}\right\} / p
$$

converges to zero a.e., as $m \rightarrow \infty$, for any choice $0<p<\infty$. An $n$-dimensional version of this result was announced by Sarăsenidze [1976] for functions in $L\left(\log ^{+} L\right)^{n-1}$. Apparently, the $n$-dimensional version of (13) fails to converge a.e. to zero for certain $f \in L\left(\log ^{+} \log ^{+} L\right)^{n-1}$ for $n \geq 2$.

Several authors have considered weaker versions of (13). For example, Cybertowicz [1976] showed that if $r>0$ and if $a_{n-1, k}=\left(\begin{array}{c}n-2+r-k \\ r-1\end{array}\right) /\left(\begin{array}{c}n-1+r \\ r\end{array}\right)$ for $k \leq n-1$ and $a_{n-1, k}=0$ for $k>n-1$, then

$$
\left\{\sum_{k=0}^{n-1} a_{n, k}\left|w_{k}[f]-f\right|^{2}\right\}^{1 / 2} \rightarrow 0
$$

a.e., as $n \rightarrow \infty$ for all $f \in L^{l}$. Śarašenidze [1973] proved that given any positive, regular method of summability $\left[a_{n, k}\right]$ with $a_{n, k+1} \leq a_{n, k}$ and given any continuous $f$ there exist numbers $r_{n} \uparrow \infty$ such that

uniformly, as $n \rightarrow \infty$.

$$
\left\{\sum_{k=0}^{n-1} a_{n, k}\left|w_{k}[f]-f\right|^{r_{n}}\right\} / r_{n} \rightarrow 0
$$

Yano [1951b] investigated the growth of $(C, \beta)$ sums of Walsh-Fourier series in $L^{p}$ norms. He proved that if $1 \leq P \leq \infty,-0<\alpha<1$ and $f \in \operatorname{Lip}\left(\alpha, L^{p}\right)$ then

$$
\left\|\sigma_{n}^{\beta}(f)-f\right\|_{L^{p}}=O\left(n^{-\alpha}\right) \text {, as } n \rightarrow \infty \text {. }
$$

provided $\beta>\alpha$. Remarkably, Skvorcov [1981a] showed that this estimate holds even for $0<\beta \leq \alpha$. Moreover, he obtained an order estimate for the limiting case $\alpha=1$ : if $f \in \operatorname{Lip}\left(1, L^{p}\right)$ then

$$
\left\|\sigma_{n}^{\beta}(f)-f\right\|_{L} p=0(\log n / n) \text {, as } n \rightarrow \infty .
$$

In fact, he proved much more. He showed that there is an absolute constant $C$ such that if $n \geq 0, B>0$ and $f \in L^{p}$ then

$$
\left\|\tilde{\sigma}_{n}^{\beta}(f)-f\right\|_{L^{p}} \leq C 2^{-m} \sum_{k=0}^{m} 2^{k} \dot{\omega}_{p}\left(2^{-k}, f\right),
$$

where $m$ is defined by $2^{m} \leq n<2^{m+1}$ and $\stackrel{\sim}{\sigma}_{n}^{\beta}(f)$ represents the partial $(C, \beta)$ sum of $W[f]$ in any piecewise linear rearrangement (see $\S 3$ ). Thus the order estimates above hold for Walsh-Kaczmarcz series as well as Walsh (-Paley) series. In particular if $f \in L^{p}, 1 \leq p \leq \infty$, then the $(C, B)$ sum of any piecewise linear rearrangement of $W[f]$ converges to $f$ in $L^{p}$ norm. Skvorcov [1982] has generalized these results to Vilenkin 
groups of bounded type.

For results concerning $(C, \beta)$ sums of Walsh-Fourier series when $B<0$, see Tevzadze [1981].

Bicadze [1979] has obtained necessary and sufficient conditions for a multiple Walsh series with monotone coefficients in the sense of Hardy to be $(C, 1)$ summable to $f$ in $L^{p}$ norm, $0<p<1$.

Finally, Tateoka [1978] has studied localization on the unit square. Recall that if $X$ is a collection of integrable functions then a summability method $T$ has the localization property for $X$ if given any $f \in X$ which vanishes on an open set $V$, the double Walsh-Fourier series of $f$ is uniformly summable $(T)$ to zero on compact subsets of $V$. Tateoka shows that square partial sums do not have the localization property for $\mathscr{C}$, that square Abel means have the localization property for $L^{p}, p \geq 2$, but do not have it for $L^{p}, 1 \leq p<2$, and that rectangular Abel means have the localization property for $\mathscr{C}$ but not for $L^{p}, p>1$.

8. Adjustment on sets of small measure to enhance convergence. A celebrated result of Menshov for trigonometric series is that given an a.e. finite-valued, measurable $f$ and $a n \epsilon>0$ there exists a continuous function $\tilde{f}$ which coincides with $f$ off a set of measure less than $\epsilon$ such tinat the Fourier series of $\tilde{f}$ converges uniformly on $[0,2 \pi]$. Kotljar [1966] showed that this result is also true for Walsh-Fourier series.

Price [1969] gave a new Walsh proof of Menshov's theorem, utilizing the fact that the Walsh functions are characters of the dyadic group. This proof was adapted to Vilenkin groups of bounded type by Onneweer [1971c].

Concerning whether adjustment can be made to improve the decay of Walsh-Fourier coefficients, 0levski $\breve{i}$ [1978] showed that there is an $f \in \mathscr{C}$ such that given any $\tilde{f} \in L^{1}$ which coincides with $f$ on a set of positive measure, it is the case that

$$
\sum_{n=0}^{\infty}\left|a_{n}(\tilde{f})\right|^{p}=\infty \text { for all } p<2 \text {. }
$$

In particular, a continuous function cannot be adjusted so that its walsh-Fourier series is absolutely convergent. (Guličev [1979a] has also given a proof of this corollary to 0levskij's theorem.)

Adjustments can be made which result in a.e. and $L^{\top}$ convergence. Indeed, Heladze [1977] has given an outline of a proof which establishes that given $f \in L^{1}$ 
and given $\epsilon>0$ there is a measurable s with $m\{x: s(x) \neq 1\}<\epsilon$ such that $W[s f]$ converges a.e. and in $L^{1}$ norm.

Kemhadze [1977] obtained an adjustment theorem for functions $f$ continuous on the n-dimensional hypercube. He proved that given $\epsilon>0$ there is a $g$ which coincides with $f$ off a set of measure less than $\in$ such that the rectangular partial sum of the multiple Walsh-Fourier series of $f$ converges everywhere. He also shows that $g$ can be chosen so that its Walsh-Fourier series has certain prescribed gaps.

\section{APPROXIMATION BY WALSH SERIES}

9. Walsh series with gaps. The study of gap Walsh series predates the study of Walsh series since the Rademacher functions were introduced before the Walsh functions.

Coury [1974b] investigated differentiability of Rademacher series. He showed that a Rademacher series is either a.e. differentiable or almost nowhere differentiable. Using an earlier result of Balašov [1965], who made several foundational contributions to this problem, Coury proved that if a Rademacher series is differentiable at one point then it is of bounded $p$ th variation for all $p>1$. A deeper result he obtained is that any a.e. differentiable Rademacher series is of bounded variation. (Coury [1974a] has identified several conditions on the coefficients of a given Walsh series $W$ sufficient to conclude that $W$ is a.e. differentiable.)

For results about dyadic differentiability of Rademacher series see $\S 15$.

Zotikov [1976] has studied the problem of convergence of Rademacher series in Vilenkin groups $G$. He shows that the Rademacher system $\left\{R_{n}\right\}_{n=0}^{\infty}$ is independent and that if $G$ is of bounded type then

$$
\sum_{n=0}^{\infty} a_{n} R_{n}
$$

is not a.e. summable by any $T^{*}$ method (see Bary [1964]) when $\left[a_{n}^{2}=+\infty\right.$. When $G$ is of bounded type, he shows that (14) converges absolutely if and only if

$$
\sum_{n=0}^{\infty} \frac{\left|a_{n}\right|}{\sqrt{p_{n}}}<\infty
$$

in which case the limit $F$ of (14) is bounded, is continuous on $\left\{p_{n}\right\}$-adic irrationals, and has Vilenkin-Fourier series (14).

A Walsh series $\sum_{k=1}^{\infty} a_{n_{k}} w_{n_{k}}$ is called lacunary if $n_{k+1} / n_{k} \geq q>1$ for $k=1,2, \ldots$ 
Clearly every Rademacher series is a lacunary Walsh series. Coury [1973] proved that if $E$ is a set of second category which has the property of Baire and if $W$ is a lacunary Walsh series which converges to zero (or is constant) on $E$, then $W$ is actually a Walsh polynomial. A related result announced by Ebralidze [1976] is the following. Let $\left\{R_{m}, k\right\}(m=1,2, \ldots, k=1,2, \ldots)$ be a sequence of real numbers which satisfies $R_{m, k} \rightarrow 1$ as $m \rightarrow \infty$ for $k \geq 1$ and

$$
2_{2^{n}<k \leq 2^{n+1}}^{\max }\left|R_{m, k}\right|<c 2_{2^{n-1}<k<2^{n}}^{\min }\left|R_{m, k}\right|
$$

for $m \geq 1, n \geq 0$, where $c$ is a fixed, finite number $\geq 1$. If either the limit supremum or the limit infimum, as $m \rightarrow \infty$ of the sums

$$
\sum_{k=1}^{\infty} R_{m, k} a_{k} r_{k}(x)
$$

is finite for all $x$ in a set $E$ of the second category, then $\left[\left|a_{k}\right|<\infty\right.$. In the case when $E$ is an interval and $R_{m, k} \equiv 1$, this result had been obtained for any lacunary Walsh series by Morgenthaler [1957].

Concerning whether the sequence of coefficients of a Rademacher series belong to any $\ell^{p}$ space, $1<p<2$, Rodin and Semjonov [1975] have examined analogues of Khinchin's inequality for certain symmetric Banach spaces including those of Lorentz, Marcinkiewicz, and Orlicz. A corollary of their work is that a Rademacher series $f=\sum_{k=0}^{\infty} a_{k} r_{k}$ has coefficients $\left\{a_{k}\right\} \in \ell^{p}$ for some $1<p<2$ if and only if

$$
\int_{0}^{\infty}\left|y /\left(1+\log _{2}\left(1 / \lambda_{f}(y)\right)\right)\right|^{p-1} d y<\infty .
$$

Kolmogorov first noticed that convergence of a Rademacher series on a set of positive measure is sufficient to conclude that its coefficients belong to $\ell^{2}$. Morgenthaler [1957] showed this result also holds for lacunary Walsh series. Gapoškin [1971] weakened the lacunary condition considerably and Miheev [1979] pushed this result even further. He proved that if $\left[a_{k} w_{n_{k}}\right.$ is $T^{\star}$ summable on a set of positive measure, if $n_{k}+n_{l} \rightarrow \infty$ as $k, l \rightarrow \infty$ with $k>\ell$, and if for some $p>2$ there is a constant $C$ such that

$$
\left\|\sum_{k=1}^{\ell} b_{k} w_{n_{k}}\right\|_{p} \leq c\left\|\sum_{k=1}^{\ell} b_{k} w_{n_{k}}\right\|_{2}
$$

holds for $\ell \geq 1$ and any choice of real $b_{k}$ 's, then

$$
\sum_{k=1}^{\infty} a_{k}^{2}<\infty
$$


Morganthaler [1957] proved a central limit theorem for lacunary Walsh series. The idea was that a lacunary Walsh series is close to being a Rademacher series and thus might behave like independent identically distributed random variables. This approach was developed further in a series of papers in which central limit theorems, laws of iterated logarithm, and functional laws of the iterated logarithm were obtained (see Földes [1972], [1975], Berkes [1974], Takahashi [1975], and Ôhashi [1979].) For example, a walsh series $\left[a_{k} w_{n_{k}}\right.$ is called weakly lacunary if there exist $c>0$ and $0 \leq \alpha<1 / 2$ such that $n_{k+1} / n_{k}>1+c k^{-\alpha}$ for $k=1,2, \ldots$ Suppose for the remainder of this paragraph that the coefficients of a weakly lacunary Walsh series satisfy

$$
A_{N} \equiv\left(\sum_{k=1}^{N} a_{k}^{2}\right)^{1 / 2} \rightarrow+\infty \text {, as } N \rightarrow \infty \text {. }
$$

Takahashi [1975] showed if $a_{n}=0\left(A_{N} /\left[N^{\alpha}\left(\log A_{N}\right) \frac{1+\epsilon}{2}\right]\right)$, as $N \rightarrow \infty$, for some $\epsilon>0$, then

$$
\limsup _{N \rightarrow \infty}\left(2 A_{N}^{2} \log \log A_{N}^{2}\right)^{-1 / 2} \sum_{k=1}^{N} a_{k} w_{n_{k}}=1 \text { a.e.; }
$$

Földes [1975] showed if $a_{N}=o\left(A_{N} / N^{\alpha}\right)$, as $N \rightarrow \infty$, then

$$
\lim _{N \rightarrow \infty} m\left\{x: A_{N}^{-1} \sum_{k=1}^{N} a_{k} w_{n_{k}}(x) \leq y\right\}=\frac{1}{\sqrt{2 \pi}} \int_{-\infty}^{y} e^{-t^{2} / 2} d t,-\infty<y<\infty .
$$

Ôhashi [1979] obtained a local version of (15) and used it to prove that there exist weakly lacunary Walsh-Fourier series which diverge a.e. He also showed that the Földes growth condition is best possible. Specifically, he proved that given any $c>0$ and $0 \leq a, 1 / 2$ there exist integers $n_{1}<n_{2}<\ldots$ and coefficients $a_{1}, a_{2}, \ldots$ such that $n_{k+1} / n_{k}>1+c k^{-\alpha}$ for $k \geq 1$, such that $A_{N} \rightarrow \infty$, as $N \rightarrow \infty$, and such that $a_{N}=O\left(A_{N} / N^{\alpha}\right)$, as $N \rightarrow \infty$, but such that (15) fails to hold.

As noted above, under certain conditions (e.g., convergence to zero on an interval) the only lacunary Walsh series ure Walsh polynomials. Such a condition was obtained by Roider [1969] for gap Walsn. Fourier series. If $k=2^{n_{1}} 1+\ldots+2^{n_{\gamma}}$ is a positive integer with $n_{1} \geq n_{2} \geq \cdots \geq n_{\gamma} \geq 0$, the Vielfalt of $k$ is defined to be $V(k)=\gamma$. Thus a rather mild lacunary condition is given by

$$
a_{k}(f)=0 \text { for } v(k)>v
$$

for some fixed integer $\nu$. Roider proved that if $f \in L^{1}$ assumes only finitely many, or only integral, values, and if (16) is satisfied for some $v \geq 0$, then $f$ is actually 
a Walsh polynomial. For related results see Gruber [1977/78].

For a local. condition sufficient to conclude that a gap series is a Walsh polynomial see [1982] by the author. For results concerning summability of gap series see $\S 11$.

10. The Walsh system as a basis. Recall that a system $\left\{f_{1}, f_{2}, \ldots\right\}$ is a basis in some Banach space $B$ if given $f \in B$ there is a unique series $\sum_{n=1}^{\infty} a_{n} f_{n}$ which converges in $B$ norm to $f$. The Walsh system is a basis for $L^{p}, 1<p<\infty$. Kazarjan [1978] examined whether a subsystem $\left\{w_{n_{k}}\right\}_{k=1}^{\infty}$ of the walsh system can be multiplicatively completed, i.e., whether there exists a measurable $\phi$ such that $\left\{\phi(x) w_{n_{k}}(x)\right\}_{k=1}^{\infty}$ is a basis for the spaces $L^{p}, 1<p<\infty$. He proved that such a $\phi$ never exists no matter how few and far between the gaps are.

Two sys tems $\left\{f_{1}, f_{2}, \ldots\right\}$ and $\left\{g_{1}, g_{2}, \ldots\right\}$ are equivalent bases in a Banach space $B$ if given coefficients $a_{1}, a_{2}, \ldots$ the series $\left[a_{n} f_{n}\right.$ and $\left[a_{n} g_{n}\right.$ are equiconvergent in B. Ciesielski and Kwapién [1979] proved that the Walsh system and the bounded polygonals are equivalent bases in $L^{p}, 1<p<\infty$. On the other hand, Young [1976b] showed that the trigonometric and walsh systems are not equivalent bases in $L^{p}, p \neq 2$. Hence they will have different multipliers, and multipliers for the Walsh system should be investigated.

Shirey [1973] examined the Walsh system as a quasi-basis, and proved that given any set $E$ of positive measure, the quasi-basis for $L^{p}(E), 1<p<\infty$, obtained by restricting the Walsh system to $E$, is a conditional quasi-basis.

11. Approximation by Walsh series. Skvorcov [1973b] has shown that unlike the trigonometric case or the one-dimensional Walsh case, convergence of a double Walsh series $W$ on a set of positive measure is not sufficient to conclude that the coefficients of $W$ converge to zero. However, he proved that if the retangular partial sums of a double Walsh series $W$ converge on any dyadic irrational across (i.e., on a set of the form $\{a\} \times(0,1) \cup(0,1) \times\{b\}$, where $a$ and $b$ are dyadic irrationals $)$ then the coefficients of $W$ satisfy $a_{n, m} \rightarrow 0$, as $m+n \rightarrow \infty$.

One of Menshov's celebrated results is that given any measurable, a.e. finitevalued function $\phi$ there is a trigonometric series which converges to $\phi$ a.e. His techniques apply equally well to Walsh series. Thus Walsh series can be used to 
approximate measurable functions which are finite a.e.

Talaljan [1960] has shown that given any $L^{p}$ basis, $p>1$, and any measurable $\phi$ (finite or not) there is a series with respect to that basis which converges in measure to $\phi$. Thus to every measurable $\phi$ there corresponds at least one Walsh series which converges to $\phi$ in measure. This result does not hold if "in measure" is replaced by "a.e.". In fact, Talaljan and Arutunjan [1965] proved that there is no Walsh series $W$ which satisfies $W_{2} m \rightarrow+\infty$ on a set of positive measure, as $m \rightarrow \infty$. Thus Walsh series cannot be used for a.e. approximation of general measurable functions.

It is natural to ask whether rearranged walsh series or $(C, 1)$ partial sums of Walsh series can be used for a.e. approximation of measurable functions. The answer to the second question is yes, and there is strong evidence that the first question can also be answered in the affirmative. Šaginjan [1979] showed that given any positive regular method $T^{\prime}$ of summability there exist walsh series $W=\sum_{k=1}^{\infty} a_{k} w_{n}$ with $\left\{a_{k}\right\} \in \ell^{2+\epsilon}$ for $\epsilon>0$ and $\sum_{k=1}^{\infty} n_{k}^{-1}<\infty$ such that given any measurable $\phi$ there exists a subseries of $W$ which is a.e. $T^{\prime}$ summable to $\phi$. Thus given a measurable $\phi$, there exist walsh series which are a.e. $(C, \alpha)$ summable, $\alpha>0$, and a.e. Abel summable to $\phi$. And, Ovsepjan [1973] proved that there exists a walsh series which has a rearrangement that diverges a.e. to to.

This question of divergence has been addressed for double Walsh series. Kemhadze [1969] proved that if $W$ is a double Walsh series then $W_{2^{n}}, 2^{m}$ cannot diverge to to on a set of positive measure, as $n, m \rightarrow \infty$. Thus even rectangular sums of double Walsh series cannot be used to a.e. approximate measurable functions unless the functions are finite-valued a.e.

Pogosjan [1980] announced results concerning a.e. summability to to of the series

$$
T=\sum_{k=0}^{\infty} \gamma_{k} \tilde{w}_{k} / \sqrt{k+T}
$$

where $\gamma_{k}=0$, $\doteq 1$ and $\tilde{w}_{k}$ is a dyadic block rearrangement on the walsh function, i.e. $\left\{\tilde{w}_{k}: 2^{n} \leq k<2^{n+1}\right\}=\left\{w_{k}: 2^{n} \leq k<2^{n+1}\right\}$

for $n=0,1, \ldots$. A consequence of his results is that if $n_{1}<n_{2}<\ldots$ satisfies $\limsup _{k \rightarrow \infty} n_{k+1} / n_{k}=\infty$, then one can choose numbers $\gamma_{k}$ such that $T_{n_{k}} \rightarrow+\infty$ a.e. as $k \rightarrow \infty$. 
On the other hand, if the ratios $n_{k+1} / n_{k}$ are uniformly bounded, then $T_{n_{k}}$ cannot diverge to to on a set of positive measure.

Šainjan has obtained several conditions sufficient to conclude that

$$
f(x)=1 / 2\left[\limsup _{k \rightarrow \infty} S_{n_{k}}(x)+1 \operatorname{iminf} S_{n_{k}}(x)\right]
$$

holds for a.e. $x$ in some given measurable set $E$. For example, in [1974] he showed that if $S=\sum a_{k} \tilde{w}_{k}$ is some dyadic block rearrangement of a walsh series, if $S_{2} n(x)$ converges to a finite measurable $f$ on $E$, and if $\liminf _{k \rightarrow \infty} S_{n_{k}}(x)>-\infty$ for $x \in E$ then (17) holds for a.e. $x \in E$. In [1981] he proved that if $S=\sum a_{j} w_{k_{j}}$ is a gap walsh series with $k_{1}<k_{2}<\ldots, 1-k_{j} / k_{j+1}=0(1 / j)$ as $j \rightarrow \infty$ and

$$
\sum_{j=1}^{n-2} j\left|2 k_{j+1}-k_{j+2}-k_{j}\right|=0\left(k_{n}\right) \text {, as } n \rightarrow \infty,
$$

if $S$ is $(C, 1)$ summable a.e. on $E$ to a measurable, a.e. finite-valued function $f$, and if $\liminf _{k \rightarrow \infty} S_{n_{k}}(x)>-\infty$ for $x \in E$, then (17) holds a.e. on $E$. Notice that this result includes gaps of the form $k_{j}=n \cdot j^{m}$ for fixed $n, m \geq 1, j=1,2, \ldots$ Concerning the (C, 1) analogue of (17), Śaginjan [1974] proved that if $S$ is a dyadic block rearrangement of a Walsh series, if $\sigma_{2} n(x, S)$ converges, as $n \rightarrow \infty$, to a finite-valued, measurable $f$ on $E$ and if $\liminf _{k \rightarrow \infty} \sigma_{n_{k}}(x, S)>-\infty$ for $x \in E$, then

$$
f(x)=1 / 2\left[\limsup _{k \rightarrow \infty} \sigma_{n_{k}}(x, s)+\liminf _{k \rightarrow \infty} \sigma_{n_{k}}(x, s)\right]
$$

holds for a.e. $x \in E$. Contained in his proof is verification of the following "Tauberian" theorem. If $\limsup _{n \rightarrow \infty}\left|\sigma_{2 n}(x, S)\right|<\infty$ holds for all $x \in E$, then $S_{2^{n}}(x)$ converges a.e. on $E$, and

holds for a.e. $x \in E$.

$$
\liminf _{n \rightarrow \infty} \sigma_{2^{n}}(x, S) \leq \lim _{n \rightarrow \infty} S_{2^{n}}(x) \leq \limsup _{n \rightarrow \infty} \sigma_{2^{n}}(x, S)
$$

Concerning structure of sets on which Walsh series diverge, Lukašenko [1978] showed that given any $\Psi_{\delta}$ - set $E$ there is a Walsh series $W$ such that

$$
E=\left\{x: \lim _{n \rightarrow \infty} W_{n}(x) \text { does not exist }\right\} \text {. }
$$

He also proved that this result does not necessarily hold if " $\mathscr{G}_{\delta}$-set" is replaced by "F $\sigma$-set."

For every $f \in L^{p}, 1 \leq p<\infty$, let $E_{n}^{(p)}(f)$ denote the infimum of the expression $\left\|f-\sum_{k=0}^{n-1} a_{k} w_{k}\right\|_{p}$ as $\left\{a_{k}\right\}$ take on all real values, for $n=1,2, \ldots$ Golubov [1972] 
has obtained many sharp estimates relating the "non-dyadic" moduli of continuity

$$
\bar{\omega}_{p}(\delta, f) \equiv \sup _{0 \leq h \leq \delta}\left\{\int_{0}^{1}|f(x+h)-f(x)|^{p d x}\right\}^{1 / p}, 0 \leq \delta \leq 1,
$$

to the sequences $E_{n}^{(p)}(f)$. He proved that

$$
\bar{\omega}_{p}(1 / n, f) \leq 96 n^{-1 / p} \sum_{k=1}^{n} k^{1 / p-1} E_{k}^{(p)}(f)
$$

holds for all $f \in L^{p}$ and all $n \geq 1$. He also obtained the following analogues of trigonometric inequalities due to Ul'janov. If $1 \leq p<q<\infty$ there exists a constant $C$ depending only on $p$ and $q$ such that

and

$$
\|f\|_{q} \leq c\left\{\|f\|_{p}+\left[\sum_{k=1}^{\infty} k^{\frac{q}{p}-2}\left[E_{k}^{(p)}(f)\right]^{q}\right]^{1 / q}\right\}
$$

$$
E_{n}^{(q)}(f) \leq C\left\{E_{n}^{(p)}(f) n^{1 / p-1 / q}+\left[\sum_{k=n+1}^{\infty} k^{q / p-2}\left[E_{k}^{(p)}(f)\right]^{q}\right]^{1 / q}\right\}
$$

hold for $n \geq 1$, and $f \in L^{p}$. (See Golubov [1970], p. 719, for ramifications).

Siddiqi [1971] proved that given a quasi-convex sequence $\left\{a_{k}\right\}$, the Walsh series $W=\sum a_{k} w_{k}$ converges in $L^{1}$ norm if and only if $a_{k} \log k \rightarrow 0$, as $k \rightarrow \infty$.

N. WALSH-FOURIER COEFFICIENTS

12. Growth of Walsh-Fourier coefficients. Several authors have investigated conditions under which the series

$$
\sum_{k=1}^{\infty} k^{\gamma}\left|a_{k}(f)\right|^{\beta}
$$

converges for various choices of $\gamma$ and $\beta$. A unified treatment of this problem (not only in the Walsh case, but for Vilenkin groups of bounded type as well) has been given by McLaughlin [1973]. His article contains an extensive bibliography and is nearly a compendium of what is known about this problem. His main result, still the most general of its kind, is that if $1 \leq p \leq 2$, if $0<\beta \leq q$ and if $1 / p+1 / q=1$, then (18) converges when

$$
\sum_{k=1}^{\infty} k^{\gamma-\beta / q}\left|\bar{\omega}_{p}(1 / k, f)\right|^{\beta}<\infty .
$$

Ladhawala [1976] showed that (18) converges for $\gamma=-1$ and $\beta=1$ when $f$ belongs to dyadic $H^{1}$. His proof contains the following interesting fact. If $\left\{a_{k}\right\}$ is a sequence of real numbers which satisfies $a_{k}=0(1 / k)$, as $k \rightarrow \infty$, then $a_{k}=a_{k}(\phi)$ for some $\phi$ of bounded mean oscillation. Chao [1981] has obtained these results for Vilenkin groups of bounded type. In addition, Quek and Yap [1980] have shown that 
(18) converges for $\gamma=0$ when $f \in \operatorname{Lip}(\alpha, p)$ on any Vilenkin group of bounded type provided $\beta>p /(\alpha p+p-1)$.

Moricz [1981] has studied integrability of a Walsh series with monotone coefficients $a_{0} \geq a_{1} \geq \cdots \geq a_{k} \geq 0$. He proved that for $r>1, \beta=r$, and $\gamma=r-2$, the series (18) converges if and only if

$$
\left.I_{r} \equiv \int_{0}^{1} \sup _{n \geq 0}\left|\sum_{k=0}^{n} a_{k} w_{k}(x)\right|\right)^{r} d x<\infty .
$$

In the case $r=1$, it turns out that $\sum a_{k} / k<\infty$ is both necessary and sufficient for $I_{r}$ to be finite.

Vilenkin and Rubinštein [1975] have several estimates of the tails of (18) when $\gamma=0$ in the Vilenkin group setting. They proved that if $f \in L^{2}(G)$ then

$$
\left\{\sum_{j=m_{k}}^{\infty}\left|a_{j}(f)\right|^{2}\right\}^{1 / 2} \leq 1 / \sqrt{2} \omega_{k}^{(2)}(f), k=1,2, \ldots .
$$

They obtained analogues of two theorems of Lorenz for Vilenkin groups of bounded type: if $p_{k} \leq C$ for $k=1,2, \ldots$, if $f \in \operatorname{Lip} \alpha(G), \alpha>1 / p-1 / 2$ for some $0<p \leq 2$, then

$$
\left\{\sum_{j=m_{k}}^{\infty}\left|a_{j}(f)\right|^{p}\right\}^{1 / p} \leq C m_{k} 1 / p-\alpha-1 / 2 .
$$

And, if $\sum_{j=m_{k}}^{\infty}\left|a_{j}(f)\right| \leq C m_{k}^{-\alpha}$ for some $\alpha>0$ then $f \in \operatorname{Lip} \alpha(G)$.

Horoško [1972] considered the problem of determining exact estimates for the growth of Walsh-Fourier coefficients of functions belonging to $H_{V}$, the class of $f$ whose variation does not exceed the constant $V>0$. He proved that the maximum of

$$
\sup _{f \in H_{V}}\left|a_{k}(f)\right|
$$

for $2^{m} \leq k<2^{m+1}$ is $V / 2^{m+1}$ and that the minimum of (19) for $2^{m} \leq k<2^{m+1}$ is $V / 2^{m+2}$.

Rubinštein [1980] obtained an extension of Parseval's identity from $1<p<\infty$ to $p=1$ for Vilenkin groups of bounded type. He announced that if

$$
n \omega_{n}{ }^{(1)}(f) \cdot \omega_{n}(g) \rightarrow 0 \text {, as } n \rightarrow \infty \text {, }
$$

then $\int_{G} f \bar{g} d m=\sum_{k=0}^{\infty} a_{k}(f) \overline{a_{k}(g)}$. He indicated that this identity does not necessarily hold if (20) is relaxed to

$$
\liminf _{n \rightarrow \infty} n \omega_{n}^{(1)}(f) \omega_{n}(g)>0
$$

13. Conditions on Walsh-Fourier coefficients sufficient to conclude that $\underline{f}$ is 
constant. Fine [1949] was among the first to notice that the Walsh-Fourier coefficients of a smooth function cannot decay too rapidly. He showed that if $f$ is absolutely continuous and if $k a_{k}(f) \rightarrow 0$, as $k \rightarrow \infty$, then $f$ is constant on $[0,1]$.

Bočkarev [1970] sought to determine how rapidly Walsh-Fourier coefficients of continuous functions can decay. He proved that if $\left|a_{k}(f)\right| \leq d_{k}$, where $d_{k}+0$ and $\left[d_{k}<\infty\right.$ and if $f$ is continuous, then $f$ is constant on $[0,1]$. Hence the WalshFourier coefficients of a non-constant continuous $f$ cannot satisfy

$$
\left|a_{k}(f)\right|=0\left(1 /\left[k(\log k)^{\alpha}\right]\right) \text {, as } k \rightarrow \infty \text {, }
$$

for some $\alpha>1$. The condition $\alpha>1$ cannot be relaxed. Indeed, Bočkarev constructed a non-constant, continuous $f$ whose Walsh-Fourier coefficients satisfy

$$
\left|a_{k}(f)\right|=0(1 /[k \log k]) \text {, as } k \rightarrow \infty \text { (see Bočkarev [1978b], p. 19). }
$$

One drawback to Bockkarev's result is that it only applies to functions whose Walsh-Fourier series are absolutely convergent. Coury [1974a] worked to remove this restriction. He proved that if $f$ is continuous and if

$$
2^{p} \sum_{m=p}^{\infty} R_{m} \rightarrow 0, \text { as } p \rightarrow \infty
$$

where $R_{m} \equiv\left\{\left\{\left|a_{k}(f)-a_{k+1}(f)\right|: 2^{m} \leq k<2^{m+1}-1\right\}\right.$, then $f$ is constant. It follows that no non-constant, continuous $f$ whose Walsh-Fourier coefficients are monotone decreasing can satisfy $2^{n} a_{2} n(f) \rightarrow 0$, as $n \rightarrow \infty$. The author [1983] has generalized this result by weakening condition (21).

Coury has proved (but not yet published) that there is no non-constant, continuous $f$ which satisfies

$$
\sum_{k=1}^{\infty} k\left|a_{k}(f)\right|<\infty
$$

Powell and the author [1981] generalized this result: if $f$ is continuous, if $\sum_{k=0}^{\infty} k^{\alpha} a_{k}(f) w_{k}$ converges on $(0,1)$ for some $\alpha \geq 1$, with the further assumption that

$$
\lim _{n \rightarrow \infty} \sum_{j=1}^{\infty} \sum_{k=j 2^{n}}^{(j+1) 2^{n}-1} j 2^{n} a_{k}(f) w_{k}(x), x \in(0,1),
$$

exists when $\alpha=1$, then $f$ is constant.

The author [1979b] has identified several conditions sufficient to conclude that a function is constant on $[0,1]$. The most interesting one is that there is no non-constant continuously differentiable function which satisfies 


$$
\sup _{n>0} \mid \sum_{k=0}^{n} 2_{j=2^{k+1}-1}^{a_{j}(f) w_{j}(\rho) \mid<\infty}
$$

for all dyadic rationals $\rho \in[0,1]$.

v. DYADIC DIFFERENTIATION

14. The strong dyadic derivative. Butzer and Wagner [1973] introduced the dyadic derivative, which interacts with the Walsh-Paley functions in much the same way as the classical derivative interacts with the exponential functions $\left\{e^{i n x}\right\}$. Chief among these interactions is the fact that Walsh functions are the eigenfunctions of the induced differential operator, and

$$
D w_{k}=k w_{k}, k=0,1, \ldots .
$$

Butzer and Wagner [1972] also defined a derivative which yields (22) for the Walsh functions in the Kaczmarcz ordering. The Walsh-Paley definition is described below.

Let $X$ represent either the space $\mathscr{C}$ or one of the spaces $L^{p}, 1 \leq p<\infty$. A given $f \in X$ is said to be strongly dyadically differentiable in $X$ (more briefly, $X$ differentiable) if the sequence of functions

$$
d_{n}(f, x) \equiv \sum_{j=0}^{n-1} 2^{j-1}\left[f(x)-f\left(x+2^{-j-1}\right)\right]
$$

converges in the norm of $X$, in which case the limit of (23) is called the strong dyadic derivative of $f$ and denoted by $D f$. The dyadic antiderivative $I$ is defined by

$$
\text { If }(x)=\int_{0}^{1} f(x+t)\left[1+\sum_{k=1}^{\infty} k^{-1} w_{k}(t)\right] d t
$$

Butzer and Wagner [1973] proved that $D$ is a closed linear operator, that if $f \in X$ then If is $X$ differentiable with

$$
D(I f)=f \text { a.e. } .
$$

And $I(D f)=f$ a.e. when $f$ is $X$ differentiable. Thus the fundamental theorem of dyadic calculus is true. They also obtained the following characterization of strong dyadic differentiation. For any $f \in X$ the following three conditions are equivalent:

$$
\begin{aligned}
& f \text { is } X \text { differentiable and } g=D f \text {; } \\
& \text { there is a } g \in X \text { such that } \\
& k a_{k}(f)=a_{k}(g) \text { for } k=1,2, \ldots ;
\end{aligned}
$$

and 
This same characterization was extended to include $X=$ dyadic $H^{\top}$ by Ladhawala [1976]. Butzer and Wagner [1975] also obtained a closed form for the "dyadic integral" operator (24). They proved that if $f \in L^{\top}$ then

$$
\text { If }=a_{0}(f)+\sum_{k=1}^{\infty} a_{k}(f) / k \text { a.e. }
$$

Most results about the dyadic derivative have reinforced the idea that it is the correct derivative to use in Walsh-Fourier analysis. For example, Butzer and Wagner [1973] confirmed the connection between differentiability and Lipschitz spaces by showing that any $f \in \operatorname{Lip}(\alpha, X)$ for $\alpha>1$ has a strong dyadic derivative $D f \in X$, and, moreover, any function $f$ with a strong dyadic derivative $D f \in X$ necessarily belongs to Lip $(\beta, X)$ for $0<\beta \leq 1$. Ladhawala [1976] showed that If converges absolutely when $f$ belongs to dyadic $H^{1}$, i.e., if $f$ is strongly dyadically differentiable in dyadic $H^{1}$ then $W[f]$ is absolutely convergent. In particular, if Df exists in dyadic $H^{1}$ (or in any $L^{p}$ for $p>1$ ), then $f$ is continuous on the group.

On the other hand, Penney [1976] has shown that not all strongly dyadically differentiable functions are continuous on the group. In fact, Ladhawala [1976] proved that if $D f$ exists in $L^{\prime}$ then the best one can say is that $f \in B M O$; $f$ may not even be bounded.

Several authors have considered generalizations of the dyadic derivative to objects other than functions defined on $[0,1]$ with dyadic structure. Pál[1975] defined $D f$ on the dyadic field, i.e., for functions $f \in L^{1}(0, \infty)$ and showed that the "Walsh transform" $F$ (see Fine [1950]) interacts with D as it should: namely, if Df exists then $\mathscr{F}(D f)(y)=y \mathscr{F}(y)$ and $D\left(F_{f}\right)(x)=\mathscr{F}(x f(x))$ if $x f(x) \in L^{1}(0, \infty)$. In [1977] he constructed an indefinite integral for $D$ and proved a fundamental theorem of calculus in this setting.

Onneweer [1977] introduced a Vilenkin group analogue of the dyadic derivative, showing that the characters of the Vilenkin group are the eigenfunctions of the induced differential operator and argued that the Butzer-Wagner characterization (26), (27), and (28) carries over without extra work to this setting. Pâl and Simon [1977b] proved a fundamental theorem of calculus for Onneweer's derivative. We shall call this derivative "dyadic" below.

From the beginning, it has bothered some that the definition of the dyadic 
derivative depends on the ordering of the characters. Onneweer [1978b] endeavored to erase this deficiency, while at the same time coming up with a derivative whose eigenvalues represented the "frequency" of the characters as in the classical case with $d / d x$ and $\left\{e^{i n x}\right\}$. His efforts were concentrated on $p$-adic and p-series fields $K$. (Recall that the group of integers of a 2-series field is precisely the dyadic group $\left.2^{\omega}\right)$. His definition yields different eigenvalues on $2^{\omega}$ from those of the strong dyadic derivative discussed above since $D w_{k}=2^{n} w_{k}$ where $2^{n} \leq k<2^{n+1}, n=0,1, \ldots$ Nevertheless, the characters of $K$ are again eigenfunctions of the associated differential operator, which turns out to be a closed, linear operator in $L^{1}(K)$. Onneweer [1979b] introduced another "dyadic" derivative and compared all four derivatives the Butzer-Wagner derivative, the one introduced by Pál on the dyadic field, his earlier one on $K$, and this latest one defined on the dyadic group and the dyadic field. This latest derivative behaves much as the others but has the twin virtues of simplicity and the hope of lending itself more easily to an interpretation with possible applications in the physical sciences.

15. The pointwise dyadic derivative. A function $f$ defined on $[0,1]$ is said to have a dyadic derivative at a point $x$ if the sequence of real numbers $\left\{d_{n}(f, x)\right\}$ (see (23)) converges, as $n \rightarrow \infty$. In this case, we denote this limit by $\dot{d} f(x)$. This definition was introduced by Butzer and Wagner [1975], who identified a large class of functions on which the pointwise dyadic derivative and the strong dyadic derivative agree a.e. Skvorcov and the author [1979] observed that anytime $\dot{d} f(x)$ exists, the classical Dini derivatives of $f$ satisfy $D^{+} f(x) \geq 0 \geq D_{-} f(x)$. Hence it is impossible for a nonconstant, (classically) continuous function to be dyadically differentiable at all but countably many points in $(0,1)$. Since $d_{k}(x)=k w_{k}(x)$ for all $x \in[0,1]$, this impossibility does not extend to functions continuous on the group.

Schipp [1974] proved a fundamental theorem for the pointwise dyadic derivative. He showed that if $f \in L^{1}$ with $a_{0}(f)=0$ then $\dot{d}(I f)=f$ a.e. (compare with (25)). Pál and Simon [1977b] obtained this same result for the dyadic derivative on Vilenkin groups of bounded type. Schipp [1976b] extended his [1974] theorem to handle certain Stieltjes measures associated with functions of bounded variation. In all these results the method of proof is to show that the maximal operators 


$$
\sup _{n>0} d_{n}(I f, x), x \in[0,1] \text {. }
$$

are of strong type $(p, p), 1<p \leq \infty$, and of weak type $(1,1)$.

Onneweer [1977] showed that on a Vilenkin group $G$ there exist continuous $f$ which are nowhere dyadically differentiable. He also proved that a Rademacher series $R=\left[a_{k} r_{k}\right.$ is dyadically differentiable at $x$ if and only if

$$
\sum_{k=0}^{\infty} m_{k} a_{k} r_{k}(x)
$$

converges, in which case

$$
\dot{d} R=\sum_{k=0}^{\infty} m_{k} a_{k} r_{k}(x)
$$

It follows that a Rademacher series on $G$ is either a.e. or almost nowhere dyadically differentiable. Moreover, if the dyadic derivative of a Rademacher series is constant on some open subset of $G$, then that series is actually a polynomial (see also [1982] by the author). Thus the non-local property of the dyadic derivative is extreme. 16. Term by term dyadic differentiation. Butzer and Wagner [1975] were first to examine conditions under which a walsh series $\left[a_{k} w_{k}\right.$ represents a dyadically differentiable function $f$ which satisfies

$$
\dot{d} f(x)=\sum_{k=1}^{\infty} k a_{k} w_{k}(x) .
$$

This problem is completely solved for Rademacher series (see (29)) but is still open even for lacunary Walsh series.

Several authors have identified conditions sufficient for (30) to hold. Butzer and Wagner [1975] showed that (30) holds a.e. when the sequences $\left\{a_{k}\right\}$ and $\left\{k a_{k}\right\}$ are quasi-convex and $k a_{k} \rightarrow 0$ as $k \rightarrow \infty$. They also showed that (30) holds everywhere when $\left[k\left|a_{k}\right|<\infty\right.$, i.e., when the derived series is absolutely convergent.

Schipp [1976a] proved that (30) holds for all $x \neq 2^{-n}(n>0)$ when $k a_{k}+0$, as $k \rightarrow \infty$. This result was generalized by Skvorcov and the author [1979], who showed that if condition (21) holds, and if $x \neq 2^{-n}$ for $n>0$, then a necessary and sufficient condition for $\dot{d} f(x)$ to exist is that the sequence

$$
\sum_{k=0}^{2^{n}-1} k a_{k} w_{k}(x)
$$

converges in which case the limit of (31), as $n \rightarrow \infty$, equals $\dot{d} f(x)$. It follows that if $a_{k}+0$, if $2^{k} a_{2} k \rightarrow 0$, as $k \rightarrow \infty$, and if the derived series converges then (30) holds 
for $x \neq 2^{-n}(n>0)$. Also, if $k a_{k} \rightarrow 0$ and if

$$
k \sum_{j=k}^{2 k}\left|a_{j}-a_{j+1}\right| \rightarrow 0,
$$

as $k \rightarrow \infty$, then $\dot{d} f(x)$ exists if and only if (31) converges, as $n \rightarrow \infty$.

Powell and the author [1981] showed that (30) holds for any $x$ which satisfies

$$
\sum_{k=1}^{\infty} k^{\alpha} a_{k} w_{k}(x)<\infty
$$

for some $\alpha>1$, and that (30) holds for all $x \in[0,1]$ if $a_{k} \downarrow 0$, as $k \rightarrow \infty$, and $\left[\left|a_{k}\right|<\infty\right.$. They also found necessary and sufficient conditions for (30) to hold when the derived series converges. In fact, if $f(t)$ exists at $t=x$ and $t=x+2^{-n-1}$ $(n \geq 0)$ and if the right hand side of $(30)$ converges, then a necessary and sufficient condition for $\dot{d} f(x)$ to exist is that

$$
R(x) \equiv \lim _{n \rightarrow \infty} \sum_{j=1}^{\infty} \sum_{k=j 2^{n}}^{(j+1) 2^{n}-1} j 2^{n} a_{k} w_{k}(x)
$$

exist, in which case,

$$
\dot{d} f(x)=\sum_{k=1}^{\infty} k a_{k} w_{k}(x)-R(x)
$$

U. UNIQUENESS

17. Uniqueness of Walsh series with monotone coefficients. Balašov [1971] proved that if the coefficients of a Walsh-Kaczmarcz series $S$ are convex and decrease monotonically to zero, then $S$ is the Walsh-Kaczmarcz-Fourier series of some function in L'. Yano [1951a] had obtained this same result for Walsh-Paley series with "quasiconvex" replacing "convex". Coury [1974a] showed that quasi-convexity is essential to this result. Indeed he constructed a Walsh series with monotonically decreasing coefficients which converges to a non-integrable function and is not a Walsh-Fourier series.

In the trigonometric case, a series with convex coefficients which decrease monotonically to zero converges to a non-negative function. Coury [1974a] showed that this result does not hold for Walsh series. Indeed, he constructed a convex monotone sequence $\left\{a_{k}\right\}$ for which $w=\sum_{k=0}^{\infty} a_{k} w_{k}$ assumes negative values. He went on to show that if the sequence of coefficients is completely monotone, then analogy with the trigonometric case is restored and $W \geq 0$.

Siddiqi [1971] proved that if $a_{k} \downarrow 0$, as $k \rightarrow \infty$, and if $\left[\left(a_{k}-a_{k+1}\right) \log k<\infty\right.$ then 
$\left[a_{k} w_{k}\right.$ is a walsh-Fourier series.

18. Sets of uniqueness. A set $E \subseteq[0,1]$ or $2^{\omega}$ is said to be a set of uniqueness (or a $U$-set) if the zero series is the only Walsh series which converges to zero off E. Šneider [1949] showed that any countable subset of $[0,1]$ is a $U$-set and that the Cantor set formed by removing middle halves is a $U$-set. He also showed that any $U$-set has Lebesgue measure zero, but not all sets of Lebesgue measure zero are $U$-sets. Coury [1970], [1975] found a class of sets of Lebesgue measure zero which are dense in $[0,1]$ but are not $U$-sets. Skvorcov [1977b] proved that given any positive, increasing $h$ on $[0, \infty)$ with $h(0)=0$ there exists a perfect set $E \subseteq[0,1]$ whose $h$-measure is zero such that $E$ is not a $U$-set. Thus the problem of identifying those perfect subsets of $[0,1]$ which are $U$-sets is still open.

Gevorkjan [1981] has shown that given any $\epsilon_{k}+0$ there is a set $E \subseteq[0,1]$ of measure one such that $W=a_{k} w_{k}$ with $w_{2} n_{j} \rightarrow 0$ off $E$ and $\left|a_{k}\right| \leq \epsilon_{k}$ implies $W \equiv 0$.

On the dyadic group, more is known. The author [1979a] introduced a group analogue of $\mathrm{H}$-sets, and showed that every $\mathrm{H}$-set in $2^{\omega}$ is a $U$-set. Building on this, Yoneda [1982] proved that every closed subgroup of the dyadic group of Haar measure zero is a $U$-set. It follows that a large class of Cantor-like sets in the dyadic group are $U$-sets.

Lippman and the author [1980] carried the Pyatetskii-Shapiro structure theorem over to the group $2^{\omega}$. They showed that any closed $U$-set in the dyadic group is a countable union of elementary $U$-sets. An elementary $U$-set $E$ is one for which there exists a sequence of functions $f_{1}, f_{2}, \ldots$ whose Walsh-Fourier series are absolutely convergent and vanish on $E$, which converges to 1 in the weak * topology (i.e., given any pseudo-function $\Lambda, \Lambda\left(f_{n}-1\right) \rightarrow 0$, as $\left.n \rightarrow \infty\right)$. The author [1971] had earlier shown that a countable union of closed $U$-sets is again a $U$-set. It is not known whether the condition "closed" can be relaxed in either of these results.

A set $E \subseteq 2^{\omega}$ is said to be a $U$-set for a class $\not$ of Walsh series, if the hypothesis $W \in A A$, with $W_{2} n(x) \rightarrow 0$ as $n \rightarrow \infty$ for $x \notin E$, implies that $W$ is the zero series. Clearly, every $U$-set for $\mathscr{A}$ is a $U$-set. Crittenden and Shapiro [1965] proved that a Borel set $E$ is a $U$-set for the class of Walsh series which satisfy

$$
\lim _{n \rightarrow \infty} 2^{-n} w_{2} n(x \pm 0)=0 \text { for all } x \in[0,1]
$$


if and only if $E$ is countable. The author [1975] introduced classes $\mathscr{F}_{\alpha}^{+}, 0<\alpha<1$, which are smaller than the class of Walsh series which satisfy (32), and showed that a closed set $E$ is a $U$-set for $F_{\alpha}^{+}$if and only if $E$ is of $\alpha$-capacity zero.

19. Uniqueness of approximating Walsh series. Arutunjan and Talajan [1964] proved that if $W$ is a Walsh series whose coefficients $a_{k} \rightarrow 0$ as $k \rightarrow \infty$, if $f$ is a finitevalued integrable function and if there exist integers $n_{1}<n_{2}<\ldots$ such that

$$
\lim _{j \rightarrow \infty} w_{2} n_{j}(x)=f(x),
$$

for all but countably many $x \in[0,1]$, then $W$ is the Walsh-Fourier series of $f$. Skvorcov [1968] showed that this result remains true if "integrable" is replaced by "Perron integrable" and "Walsh-Fourier series" is replaced by "Perron-Walsh-Fourier series". In [1980b], however, he showed that it does not extend to functions which are Denjoy integrable in the wide sense. A multiple Walsh-Fourier series version of the result of Arutunjan and Talaljan was obtained by Movsisjan [1974].

Skvorcov has also extensively examined the problem of obtaining uniqueness with conditions weaker than (33). On the positive side, he proved [1974], [1977a] that if $W$ is a Walsh series whose coefficients tend to zero, if $n_{1}<n_{2}<\ldots$ is a sequence of integers which satisfies-either

$$
2^{j} \leq n_{j}<2^{j+1}, j=0,1, \ldots,
$$

or $V\left(n_{j}\right) \leq A \leq \infty$ (see definition above display (16)), and if

$$
\limsup _{j \rightarrow \infty}\left|W_{n_{j}}(x)\right| \leq M<\infty
$$

for all but countably many $x \in[0,1]$, then $W$ is the Walsh-Fourier series of the function $f(x) \equiv$ limsup $W_{n_{j}}(x)$, as $j \rightarrow \infty$. In [1980a] he proved that if $w$ is a Walsh series which satisfies (32) (a condition weaker than $a_{k} \rightarrow 0$ ), if $f$ is a finite valued, Perron integrable function, and if $n_{1}<n_{2}<\ldots$ satisfies $(34)$, then

$$
\lim _{j \rightarrow \infty} w_{n_{j}}(x)=f(x)
$$

for all but countably many $x \in[0,1]$ is sufficient to conclude that $W$ is the PerronWalsh-Fourier series of $f$. On the negative side, he showed [1975] that (35) is not sufficient for uniqueness to hold if $n_{1}<n_{2}<\ldots$ is arbitrary. Indeed, he constructed a non-zero Walsh series $W$ and a sequence of integers $\left\{n_{j}\right\}$ such that $W_{n_{j}} \rightarrow 0$ everywhere, as $j \rightarrow \infty$. 
Concerning uniqueness of non-convergent Walsh series, Crittenden and Shapiro. [1965] showed that if $W$ is a Walsh series which satisfies (32), if both

$$
\limsup _{n \rightarrow \infty}\left|W_{2} n(x)\right|<\infty
$$

and

$$
\liminf _{n \rightarrow \infty} w_{2} n(x) \geq f(x)
$$

hold for all but countably many $x \in[0,1]$, where $f$ is an integrable function, then $W$ is the Walsh-Fourier series of some $g \in L^{1}$. A portion of their argument which involve: a rather intricate application of the Baire category theorem has been shortened by Lindah1 [1971]. Ovsepjan [1973] showed that (36) is crucial to uniqueness here. Infact, he proved that given any continuous $f$ and any permutation $\tilde{w}_{0}, \tilde{w}_{1}, \ldots$ of the Walsh functions, there is a Walsh series $w=\sum a_{k} w_{k}$ such that $w_{n} \geq f, w_{n} \rightarrow f$ a.e., as $n \rightarrow \infty$, but $W$ is not the Walsh-Fourier series of $f$.

Skvorcov [1973a] proved that if $W$ is a Walsh series whose coefficients tend to zero, if $f$ is an integrable function, and if

$$
\liminf _{n \rightarrow \infty} w_{2} n(x) \leq f(x) \leq \limsup _{n \rightarrow \infty} w_{2} n(x)
$$

for all $x \in[0,1] \sim E$, where $E$ is a closed $U$-set, then $W$ is the Walsh-Fourier series of $f$. The author [1977] proved that if (37) holds for $x \in[0,1] \sim E$, where $E$ is a countable set, and if (32) holds for $x \in E$ and all dyadic rational $x$, then $W$ is the Walsh-Fourier series of $f$. (See [1980] by the author also).

For many years the problem of uniqueness for $(C, 1)$ summable Walsh series was open. Crittenden [1964] made some progress toward this problem, but never published his results. Skvorcov [1976b] solved the problem with an elegant proof that if $W$ is a Walsh series which satisfies (32) and if the Cesaro sums of $W$ satisfy

$$
\limsup _{n \rightarrow \infty}\left|\sigma_{2} n(x)\right|<\infty
$$

for all but countably many $x \in[0,1]$, and

$$
\lim _{n \rightarrow \infty} \sigma_{2} n(x)=f(x) \text { a.e., }
$$

for some Perron integrable $f$, then $W$ is the Perron-Walsh-Fourier series of $f$. 20. Null series. A non-zero Walsh series which converges a.e. to zero is called a null series. Thus null series provide counter examples for certain conjectures concerning uniqueness. For example, Schipp [1969b] showed that non-negative partial 
sums is not a sufficient condition for uniqueness; he constructed a null series $W$ which satisfies $W_{n} \geq 0$ for $n \geq 0$.

Coury [1973] constructed a non-zero lacunary Walsh series which converges to zero on a set of positive measure, but proved that there are no lacunary null series.

Skvorcov [1976a] proved that a null series can diverge to to on perfect sets. Thus to insure uniqueness, conditions like (36) must hold for all but countably many $x$. Skvorcov [1977c] also examined how fast the coefficients of a null series can converge to zero. By the Riesz-Fischer theorem they cannot converge to zero arbitrarily fast. He proved that given $M_{n} \uparrow \infty$ as $n \rightarrow \infty$, there exists a null series $W$ whose coefficients satisfy

$$
\sum_{k=1}^{n} a_{k}^{2} \leq M_{n}, n \geq 1
$$

He also obtained this result for Vilenkin groups of bounded type [1979].

21. Closing comments. Up to this point we have mentioned some specific questions which have not yet been answered. In this final subsection we speculate about the future in a more general way.

The study of pointwise dyadic differentiation is in its infancy. The initial idea that $\dot{d}$ kept track of zero crossings has not been exploited. Moreover, even obvious problems, such as finding conditions under which $f_{n} \rightarrow f$ implies $\dot{d}_{n} \rightarrow \dot{d f}_{\text {, }}$ remain unexplored. More information of this type could help decide whether the operator $f \rightarrow \dot{d f}$ is really "differentiation" or merely a special multiplier.

Another frontier is provided by the spaces dyadic $H^{1}$ and dyadic BMO. Questions which need addressing include: Which walsh series results known for $L^{p}, p>1$ (respectively, $p=\infty$ ) can be extended to dyadic $H^{\top}$ (respectively, dyadic BMO)? Which classical trigonometric results about $H^{1}$ and BMO have Walsh analogues? For example, if $f \in$ dyadic $H^{l}$ is of bounded variation, does $W[f]$ converge absolutely? In view of the result of Davis [1980], cited in the introductory section above, dyadic $H^{1}$ contains information about classical $H^{1}$. Garnett and Jones [1982] have used this connection to show how classical results can be obtained more easily by looking at the dyadic case first. Perhaps Walsh series will begin to provide theorems eventuating in trigonometric analogues which solve long standing problems, or in trigonometric proofs which are simpler than those now known. Specific suggestions include 
determining which perfect subsets of $[0,1]$ are sets of uniqueness for Walsh series, resolution of whether every Borel set of uniqueness for Walsh series is a set of first category, and a new proof of a.e. convergence of $L^{p}$ Walsh-Fourier series $p>1$.

Finally, work on multiple Walsh series has just begun. Earlier it was held that since $2^{\omega} \times 2^{\omega}$ is homeomorphic and isomorphic to $2^{\omega}$, nothing new would be gained by studying such walsh series. However, pulling back results from $2^{\omega} \times 2^{\omega}$ to $2^{\omega}$ via such a homeomorphic isomorphism forces a non-standard enumeration on the characters of $2^{\omega}$. Thus multiple Walsh analysis provides a new way to study rearrangements of Walsh series. Since most rearrangements which arise in this way are rather wild (not even dyadic block rearrangements), there is surely good reason to study walsh series in higher dimensions.

\section{REFERENCES}

(The numbers following "From" in the references below indicate the page(s) in this article where that reference is cited. $\stackrel{\mathrm{S}}{\mathrm{f}}$ follows $S$ in our alphabetizing scheme.) ARUTUNJAN, F.G., and TALALJAN, A.A.

[1964] On Uniqueness of Haar and Walsh Series. Izv. Akad. Nauk SSSR 28, 1391-1408. (From p. 659)

BAHŠECJAN, A.V.

[1975] On Some Generalized Systems of Rademacher and Walsh. Izv Akad. Nauk Armjan. SSR 10, 85-91. (From p. 636)

[1979] On Permutations of the Walsh System. Akad. Nauk Armjan. SSR Dokl. 69, 98-102. (From p. 636)

BAİARSTANOVA, A.S.

[1979] Simation of Subsequences of Partial Sums of Walsh-Fourier Series. iz. Akad. Nauk Kazan. SSR Fiz. - Mat. $\underline{3}$, 59-62. (From p. 641)

BALAŠOV, L.A.

[1965] Series with Gaps. Izv. Akad. Nauk SSSR 29, 631-644. (From p. 644)

[1971] Series with respect to the Walsh System with Monotone Coefficients. Sibirsk. Mat. ㄴ. 12, 25-39. (From pp. 632,635,657)

BALAŠOV, L.A., and RUBINŠTEIN, A.I.

[1970] Series with respect to the : 1 alsh System and their Generalizations. J. Soviet Math. I (1973), 727-763. (From pp. 625, 635)

BARY, N.K.

[1964] A Treatise on Trigonometric Series, tr. by Margaret F. Mullins, Pergamon Press, London, p. 11. (From p. 644)

BERKES, I.

[1974] Approximation of Lacunary Walsh Series with Brownian Motion. Studia Sci. Math. Hung. 9, $111-122$. (From p. 646) 
BICADZE, M.G.

[1979] Simple and Multiple Trigonometric Series and Series with respect to the Walsh System with Monotone Coefficients. Soobšč. Akad. Nauk Gruzin. SSR 96, 545-548. (From p. 643)

BILLARD, $P$.

[1967] Sur la Convergence Presque Partout des Séries de Fourier-Walsh des Fonctions de 1 'Espace $L^{2}(0,7)$. Studia Math. 28, 363-388. (From p. 631)

BOCKAREV, S.V.

[1970] The Walsh-Fourier Coefficients. Izv. Akad. Nauk SSSR, Ser. Mat. 34, 203-208. (From p. 652)

[7978a] A Majorant of the Partial Sums for a Rearranged Walsh System. Dok1. Akad. Nauk SSSR 239, 509-510. (From p. 634)

[1978b] A Method of Averaging in the Theory of Orthogonal Series and Some Problems in the Theory of Bases. Trudy Mat. Inst. Steklov. No. 146, 92 pp. (From pp. 625, 637, 652)

BUTZER, P.L., and SPLETTSTÖSSER, W.

[1978] Sampling Principle for Duration-Limited Signals and Dyadic Walsh Analysis. Inform. Sci. 14, 93-106. (From p. 631)

[1980] On Quantization, Truncation and Jitter Errors in the Sampling Theorem and its Generalizations. Signal Processing 2, 101-112. (From p. 631)

BUTZER, P.L., and WAGNER, H.J.

[1972] Approximation by Walsh Polynomials and the Concept of Derivative. Applic. of Walsh functions (Proc. Sympos. Naval Res. Lab., Washington, D.C.) (From p. 653)

[1973] Walsh Series and the Concept of a Derivative. Applic. Anal. 3 , 29-46. (From pp. 639,653,654)

[1975] On Dyadic Analys is Based on the Pointwise Dyadic Derivative. Analys is Math. I, 171-196. (From pp. 654, 655, 656)

CHAO, J. - A.

[1975] Lusin Area Functions on Local Fields, Pacific J. Math 59, 383-390. (From p. 630)

[1979] Conjugate Characterizations of $\mathrm{H}^{1}$ Dyadic Martingales. Math Annalen 240, 63-67. (From p. 630)

[1982a] $H^{p}$ and BMO Regular Martingales. Harmonic Analys is (Minneapolis, Minn., 1981), pp. 274-284, Lecture Notes in Mathematics, 908, Springer, Berlin. (From p. 629, 630)

[1982b] Hardy Spaces on Regular Martingales. Martingale Theory in Harmonic Analys is and Banach Spaces (Cleveland, Ohio, 1981), pp 18-28, Lecture Notes in Mathematics, 939, Springer, Berlin. (From pp. 629, 630)

CHAO, J.-A., and TAIBLESON, M.H.

[1973] A Sub-regularity Inequality for Conjugate Systems on Local Fields. Studia Math. 46, 249-257.

[1979] Generalized Conjugate Systems on Local Fields. Studia Math. 54, 213-225. (From p. 630)

CHAO, J.-A., GILBERT, J.E., and TOMAS, P.A

[1981] Molecular Decompositions in $H^{\dot{p}}$-Theory. Suppl. Rendiconti Circ. Mat. Palermo, n.1, 115-119. (From p. 630)

CIESIELSKI, Z., and KWAPIÉN, $S$.

[1979] Some Properties of the Haar, Walsh-Paley, Franklin, and the Bounded Polygonal Orthonormal Bases in $L^{\mathrm{p}}$ Spaces. Special Issue (Dedicated to Wladvs 7 aw Orlicz on the occasion of his seventy-fifth birthday) Comment. Math. 2, 37-42. (From p. 647) 
COIFMAN, R.R., and WEISS, G.L.

[1977] Extensions of Hardy Spaces and Their use in Analys is. Bull. Amer. Math. Soc. 83, 569-645. (From p. 629)

COURY, J.E.

[1970] A Class of Walsh M-sets of Measure Zero. J. Math. Analysis Appl. 31, 318-320. (From p. 658)

[1973] Some Results on Lacunary Walsh Series. Pacif. ‥ Math. 45, 419-425. (From pp. 645, 661)

[1974a] Walsh Series with Coefficients Tending Monotonically to Zero. Pacif. J. Math. 54, 1-16. (From pp. 644, 652, 657)

[1974b] On the Differentiation of Rademacher Series. Mich. Math. ․ 21, 321-336. (From p. 644)

[1975] A Class of Walsh M-sets of Measure Zero (Corrigendum). J. Math. Analysis and Appl. 52, 669-671. (From p. 658)

CRITTENDEN, R.B.

[1964] A Theorem on the Uniqueness of the $(C, 1)$ Summability of Walsh Series. Doctoral Dissertation, University of Oregon. (From p. 660)

CRITTENDEN, R.B., and SHAPIRO, V.L.

[1965] Sets of Uniqueness on the Group $2^{\omega}$. Ann. Math. 81, 550-564. (From pp. $658,660)$

CYBERTOWICZ, $Z$.

[1976] On Strong (C, r) - Summability of Walsh-Fourier Series. Funct. Approx. Comment. Math. 3, 37-46. (From p. 642)

[1978] Some Results Concerning the Double Walsh System. Funct. Approx. Comment. Math. $\underline{6}, 87-95$.

DAVIS, B.J.

[1980] Hardy Spaces and Rearrangements. Trans. Amer. Math. Soc. 261, 211-233. (From pp. 629, 661)

EBRALIDZE, A.D.

[1976] Haar and Rademacher Series. Sobšč. Akad. Nauk Gruz. SSR 83, 297-300. (From p. 645)

FINE, N.J.

[1949] On the Walsh Functions. Trans. Amer. Math. Soc. 65, 372-414. (From pp. $637,640,652$ )

[1950] The Generalized Walsh Functions. Trans. Amer. Math. Soc. 69, 66-77. (From p. 654)

[1955] Cesaro Summability of Walsh-Fourier Series. Proc. Nat. Acad. Sci. USA 41, 588-591. (From p. 641)

FÖLDES, ANTONIA

[1972] Further Statistical Properties of the Walsh Functions. Studia Sci. Math. Hung. 7, 147-153. (From p. 646)

[1975] CentraT Limit' Theorems for Weakly Lacunary Walsh Series. Studia Sci. Math. Hungar. 10, 141-146. (From pp. 646)

GAPOŠKIN, V.

[1971] Lacunary Series with Respect to Multiplicative Systems of Functions. Sibirsk. Mat. Z 12, 295-314. (From p. 645)

GARNETT, J.B., and JONES, P.W.

[1982] BMO from Dyadic BMO. Pacific J. Math. 99, 351-371. (From p. 661)

GARSIA, A.M.

[1973] Martingale Inequalities, W.A. Benjamin, Inc., Reading Massachusetts, pp. 1-58. (From p. 629) 
GEVORKJAN, G.G.

[1981] Uniqueness Sets for Haar and Walsh Systems. Akad. Nauk Armjan. SSR Dok1. 73, 91-96. (From p. 658)

GOGOLEVA, I.M., and LOKOT', V.V.

[1979] Projection Operators with Minimal Norms with Respect to the Walsh System in the Space $L$. Applications of Funct. Anal. in Approx. Theory, Kalinin Gos. Univ., Kalinin, 158, 10-22.

GOLUBOV, B .

[1970] Series with Respect to the Haar System. J. Soviet Math I (1973), 704-726. (From p. 650)

[1972] Best Approximation of Functions in the $L^{p}$ Metric by Haar and Walsh Polynomials. Mat. Sbornik (N.S.) 87, 254-274. (From p. 649)

GOSSELIN, J

[1973] Almost Everywhere Convergence of Vilenkin-Fourier Series. Trans. Amer. Math. Soc. 185, 345-370. (From p. 632)

[1975] A Weighted Norm Inequality for Vilenkin-Fourier Series. Proc. Amer.

[1979] Math. Soc. $\frac{49}{\text { On the }}$ Convergence of Walsh-Fourier Series for $L^{2}[0,1]$, Studies in Analys is, Advances in Math., Suppl. Studies 4, Academic Press, New York, 223-232. (From p. 631)

GOSSELIN, J., and YOUNG, WO-SANG

[1975] On Rearrangements of Vilenkin-Fourier Series Which Preserve a.e.

Convergence. Trans. Amer. Math. Soc. 209, 157-174. (From p. 635)

GRUBER, H.

[1977/78] Differenzeneigenschaften von Funktionen Endlicher Vielfalt in der

Walsh-Fourier Analys is. Applic. Anal. I, 133-145. (From p. 647) GULIČEV, N.V.

[1979a] Correction of Functions up to Absolutely Convergent Fourier Series.

Vestnik Moskov. Univ. Ser. I Mat. Meh. No $5,12-15$. (From p. 643)

[1979b] Estimates of Norms of WaTsh-fourier Sums. Dokl. Akad. Nauk SSSR 248, 22-24. (From p. 640)

[1980] Approximation to Continuous Functions by Walsh-Fourier Series. Analysis Math. $\underline{6}, 269-280$. (From p. 640)

GUNDY, R.F., and WHEEDEN, R.L.

[1974] Weighted Integral Inequalities for the Non-tangential Maximal Function, Lus in Area Integral, and Walsh-Paley Series. Studia Math. 49, 107-124. (From p. 633)

HARMUTH, H.F.

[1972] Transmission of Information by Orthogonal Functions, Springer-Verlag, Berlin. (From pp. 631,635)

HARRIS, D.C., and WADE, W.R.

[1978] Sets of Divergence on the Group $2^{\omega}$. Trans Amer. Math. Soc. 240 , 385-392. (From p. 630)

HELADZE, ̌̌.v.

[1975] The Divergence Everywhere of Fourier Walsh Series. Soobšć. Akad. Nauk Gruzin. SSR 77, 305-307. (From p. 632)

[1977] The Convergence of Fourier Series Almost Everywhere and in $L^{1}$. Dok 1 . Akad. Nauk SSSR 232, 535-537. (From p. 643)

[1978] Divergence Everywhere of Fourier Series in Bounded Vilenkin Systems. Sakharth. SSR Mecn. Akad. Math. Inst. Srom 58, 224-242. (From pp. 632, 634) 
HIRSCHMAN, I.I.

[1955] The Decomposition of Walsh and Fourier Series. Memoir Amer. Math.

Soc. №. 15, 65. (From p. 633)

HOROŠKO, N.P.

[1972] Estimation of the Supremum of Fourier Coefficients on Certain Classes of Functions with Respect to Haar, Rademacher and Walsh Systems. Teor. Funkci i Funkcional. Anal. i Prilozen Vyp. 15, 3-12. (From p. 651)

HUNT, R.A.

[1970] Almost Everywhere Convergence of Walsh-Fourier Series of $L^{2}$ Functions. Actes Congres Intern. Math. 2, 655-661. (From p. 631)

KAZARJAN, K.S.

[1978] The Multiplicative Completion of Some Incomplete Bases in $L^{p}, 1<p<\infty$. Anal. Math. 4, 37-52. (From p. 647)

KEMHADZE, G.G.

[1969] The Convergence of Haar and Walsh Series. Soobšć. Akad. Nauk Gruz. SSR 56, 37-44. (From p. 648)

[1975] A Remark on the Convergence of Spherical Partial Sums of Fourier Series of Functions of Class LP, $p>1$. Dok 1. Akad. Nauk SSSR 222, 277-280. (From p. 633)

[1977] A Theorem on the Adjustment of a Function for the Multiple Walsh System. Sakharth. SSR Mecn. Akad. Math. Inst. Srom. 55, 15-26. (From p. 644)

KOTLJAR, B.D.

[1966] Walsh Series and the Theorem of Menshov on Adjustment of Functions. Izv. Akad. Nauk SSSR 30, 1193-1200. (From p. 643)

LADHAWALA, N.R.

[1976] Absolute Summability of Walsh-Fourier Series. Pacif. ‥ Math. $\underline{65}$, 103-108. (From pp. 639, 650, 654)

LADHAWALA, N.R., and PANKRATZ, D.C.

[1976] Almost Everywhere Convergence of Walsh-Fourier Series of $H^{1}$ Functions. Studia Math. 59, 85-92. (From pp. 631, 632)

LESIN, V.V.

[1977] An Analogue of a System of Reference Functions That Correspond to Walsh Functions. Mat. Zametki 21, 485-493.

LEVIZOV, S.V.

[1980] Some Properties of the Walsh System. Mat. Zametki 27, 715-720.

(From p. 627)

LINDAHL, $R$.

[1971] A Differentiation Theorem for Functions Defined on the Dyadic Rationals. Proc. Amer. Math. Soc. 30, 349-352. (From p. 660)

LIPPMAN, G.E., and WADE, W.R.

[1980] Pseudofunctions and Uniqueness on the Group of Integers of a p-Series Field. Acta. Math. Acad. Sci. Hungar. 35, 1-12. (From p. 658)

LOKOT', V.V.

[197i] A Remark on Projection Operators with Minimal Norms with Respect to the Walsh System. Proc. Cent. Reg. Ass. of Math. Dept., №. 2, Kalinin Gos. Ped. Inst., 95-101.

LUKAŚSENKO, S. JU

[1978] On Sets of Divergence and Non-Summability of Trigonometric Series. Vestnik Moskov. Gos. Univ., Ser. Mat., No. 2, 65-70. (From pp. 633, 649) 
[1980] The Structure of Sets of Divergence of Trigonometric and Walsh Series. Dokl. Akad. Nauk SSSR 253, 528-530. (From p. 633)

MAQUSI, M.

[1981] Applied Walsh Analys is, Heyden and Son, Ltd., London. (From p. 631)

MCLAUGHLIN, J.R.

[1973] Absolute Convergence of Series of Fourier Coefficients. Trans. Amer. Math. Soc. 184, 291-316. (From pp. 639, 650)

MIHEEV, I.M.

[1979] Lacunary Subsystems of a System of Walsh Functions. Sibirsk. Mat. $\underline{\underline{Z}} . \underline{20}$, 109-118. (From p. 645)

MOON, K.H.

[1975] An Everywhere Divergent Fourier-Walsh Series of the Class $L\left(\log ^{+} \log ^{+} L\right)^{1-\epsilon}$. Proc. Amer. Math. Soc. 50, 309-314. (From p. 631)

MORICZ, F.

[1981] On Walsh Series with Coefficients Tending Monotonically to Zero. Acta Math. Acad. Sci. Hungar. 38, 183-189. (From p. 651)

MORGANTHALER, G.

[1957] Walsh-Fourier Series. Trans. Amer. Math. Soc. 84, 472-307. (From pp. MOVSISJAN, $H$. $645,646)$

[1974] The Uniqueness of Double Series in the Haar and Walsh Systems. Izv. Akad. Nauk Armjan. SSR Ser. Mat. 9, 40-61. (From p. 659)

NAKATA, $S$.

[1972] On the Divergence of Rearranged Walsh Series. Tôhoku Math. ‥ 24, 275-280. (From p. 634)

[1974] On the Divergence of Rearranged Walsh Series II. Tôhoku Math. ‥ 26, 407-410. (From p. 634)

[1979] On the Unconditional Convergence of Walsh Series. Analysis Math. 5, 201-205. (From p. 634)

NOBUHIKO, FUJII

[1979] A Maximal Inequality for $H^{1}$ Functions on a Generalized Walsh-Paley Group. Proc. Amer. Math. Soc. 77, 111-116. (From p. 641)

OHASHI, K.

[1979] Central Limit Theorems for Lacunary Walsh Series and an Application. Sec. Rep. Fac. Ed. Fukushima Univ. No. 29, 7-13. (From p. 646) OLEVSKI I, A.M.

[1975] Fourier Series with Respect to General Orthogonal Systems, SpringerVerlag, Berlin (From p. 625)

[1978] The Existence of Functions with Unremovable Carleman Singularities. Dok 1. Akad. Nauk SSSR 238. (From p. 643)

OMBE, H., and ONNEWEER, C.W.

[1981] Bessel Potential Spaces and Generalized Lipschitz Spaces on Local Fields. Functional Analys is and Approximation, Birkhauser Verlag, Basel, 129-137.

ONNEWEER, C.W.

[1970] On Uniform Convergence of Walsh-Fourier Series. Pacif. J. Math. 34, 117-122. (From p. 639)

[1971a] On Moduli of Continuity and Divergence of Fourier Series on Groups.

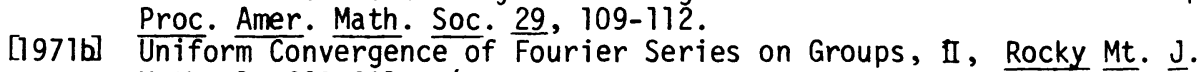
Math. 1, 623-631. (From p. 640) 
[1971c] Adjustment on Small Sets of Functions on Certain Groups. Technical Report \#225 (October 1971), University of New Mexico. (From p. 643)

[1972] Absolute Convergence of Fourier Series. Duke Math. J. 39, 599-609. (From p. 638)

[1974] Absolute Convergence of Fourier Series on Certain Groups, II. Duke Math. J. 41, 679-688. (From p. 638)

[1977] Differentiability for Rademacher Series on Groups. Acta Sci. Math (Szeged) 39, 121-128. (From pp. 654, 656)

[1978a] On L Convergence of Waish-Fourier Series. Internat. J. Math. Sci. I, 47-56. (From p. 636)

[1978b] Differentiation on a p-Adic or p-Series Field. Linear Spaces and approximation (Proc. Conf. Math. Res. Inst., Oberwolfach, 1977), 187-198, Internat. Ser. Numer. Math., vol. 40, Birkhäusen, Basel. (From p. 655)

[1979a] Sets of Divergence in the Group of Integers of a p-Adic or p-Series Field. Annales Univ. Sci. Budapest. Eötvös, Sect. Math. 21 (1978), 13-20. (From p. 634)

[1979b] On the Definition of Dyadic Differentiation. Appl. Anal. 9, 267-278. (From p. 655)

[1980] Fractional Differentiation and Lipschitz Spaces on Local Fields. Trans. Amer. Math. Soc. 258, 155-165.

ONNEWEER, C.W., and WATERMAN, D.

[1971] Uniform Convergence of Fourier Series on Groups, I. Mich. Math. ‥ 18, 265-273. (From p. 639)

[1974] Fourier Series of Functions of Harmonic Bounded Fluctuation on Groups. J. d'Anal. Math. 27, 79-93. (From p. 639)

OVSEPJAN, R.I.

[1973] The Representation of Functions by Orthogonal Series. Akad. Nauk Armjan. SSR Dok1. 57, 3-8. (From pp. 648, 660)

PÁL, J.

[1975] On the Connection Between the Concept of a Derivative Defined on the Dyadic Field and the Walsh-Fourier Transform. Annales Univ. Sci. Budapest Eötvös, Sect. Math. 18, 49-54. (From p. 654)

[1977] On the Concept of a Derivative Among Functions Defined on the Dyadic Field. SIAM J. Math. Anal. $\underline{8}, 375-391$. (From p. 654)

PÁL, J., and SIMON, P.

[977a] On a Generalization of the Concept of Derivative. Acta Math. Acad. Sci. Hungar. 29, 155-164. (From p. 641)

[1977b] On the Generalized Butzer-Wagner Type a.e. Differentiability of Integral Function. Annales Univ. Sci. Budapest. Eötvös, Sect. Math. 20, 156-165. (From pp. 654,655)

PALEY, R.E.A.C.

[1932] A Remarcable System of Orthogonal Functions. Proc. Lond. Math. Soc. 34, 241-279. (From pp. 626, 636)

PENNEY, R.

[1976] On the Rate of Growth of the Walsh Antidifferentiation Operator. Proc. Amer. Math. Soc. 55, 57-61. (From p. 654)

POGOSJAN, N.B.

[1980] Summability of Haar-Walsh Series to +o. Uspehi Mat. Nauk 35, 219-220. (From p. 648)

POWELL, C.H., and WADE, W.R.

[1981] Term by Term Dyadic Differentiation. Canad. J. Math. 33, 247-256.

(From pp. 652, 657) 
PRICE, J.J.

[1961] Orthonormal Sets with Non-negative Dirichlet Kernels, II. Trans. Amer. Math. Soc. 100, 153-161. (From p. 627)

[1969] WaTsh Series and Adjustment of Functions on Small Sets. 111. J. Math. 13, 131-136. (From p. 643)

QUEK, T.S., and YAP, L.Y.H.

[1980] Absolute Convergence of Vilenkin-Fourier Series. J. Math. Anal. Appl. 74, 1-14. (From pp. 639, 650)

[1981] Factorization of Lipschitz Functions and Absolute Convergence of Vilenkin-Fourier Series. Monat. für Math. 92, 221-229. (From pp. 638,639 )

RAM, BABU

[1975] On $L^{1}$ Convergence of a Walsh Sum. Comm. Fac. Sci. Univ. Ankara Sér. Math. 24, 9-14.

ROIDER, B.

[1969] Über Stufenfunktionen Endlicher Vielfalt. Doctoral Dissertation, University of Innsbruck, Austria. (From p. 646)

RODIN, V.A., and SEMYONOV, E.M.

[1975] Rademacher Series in Symmetric Spaces. Analys is Math. 1, 207-222. (From p. 645)

RUDIO DE FRANCIA, J.L.

[1979] Vector-valued Inequalities for Fourier and Walsh Series. Notices Amer. Math. Soc. 26, A-216.

RUBINŠTEIN, A.I.

[1978] On the Modulus of Continuity of Functions Defined on Zero-dimensional Groups. Mat. Zametki 23, 379-387. (From p. 639)

[1980] Parseval's Equality for Functions Defined on Zero-dimensional Groups. Uspehi Mat. Nauk 35, 207-208. (From p. 651)

SANADZE, D.K., and HELADZE, Š.V.

[1975] The Convergence of Multiple Walsh-Fourier Series. Soobšč. Akad. Nauk Gruz. SSR 80, 285-288. (From p. 632)

[1977] Convergence and Divergence of Multiple Fourier-Walsh Series. Sakharth SSR Mecn. Akad. Math. Inst. Srom. 55, 93-106. (From pp. 632, 634)

SCHIPP, F.

[969a] Über die Starke Summation von Walsh-Fourierreihen. Acta. Sci. Math. (Szeged) 30, 77-87. (From p. 641)

[1969b] Uber WaTsh-Fourierreihen mit Nichtnegativen Partialsummen. Annales Univ. Sci. Budapest. Eötvös, Sect. Math. 12, 43-48. (From p. 660)

[1969c] Uber die Divergenz der Walsh-Fourierreihen. Annales Univ. Sci. Budapest. Eötvös, Sect. Math. 12, 49-62. (From p. 632)

[1969d] Bemerkung zur Starken Sumation der Walsh-Fourierreihen. Acta Math. Acad. Sci. Hung. 29, 263-274.

[1973] Uber die Konvergenz von Reihen nach Produkt Systemen. Acta Sci. Math. (Szeged) 35, 13-16.

[1974] dber einen Ableitungsbegriff von P.L. Butzer and H.J. Wagner. Math Balkanica 4, 547-546. (From p. 655)

[1975] Certain Rearrangements of Series in the Walsh System. Mat. Zametki 18, 193-201. (From p. 636)

[1976a] On Term by Term Dyadic Differentiability of Walsh Series. Analysis Math. 2, 149-154. (From p. 656)

[1976b] On the Dyadic Derivative. Acta Math. Acad. Sci. Hungar. 28, 145-152. (From p. 655)

[1976c] Uber Geweissen Maximaloperatoren. Annales Univ. Sci. Budapest. Eötvös, Sect. Math. 18 (1975), 189-195. (From p. 641) 
[976d] On $L^{P}$-norm Convergence of Series with Respect to Product Systems. Analys is Math. 2, 49-64. (From p. 636)

SHIREY, J.

[1973] Restricting a Schauder Bas is to a Set of Positive Measure. Trans. Amer. Math. Soc. 184, 61-71. (From p. 647)

SIDDIQI, A.H.

[969a] On the Walsh-Fourier Coefficients of Certain Ciasses of Functions. Riv. Mat. Univ. Parma (2) 10, 157-163.

[1969b] On Lacunary Partial Sums of Walsh-Fourier Series. Rend. Circ. Mat. Palmero (2) $18,313-318$.

[1971] Wa1sh-Fourier Series with Monotone Coefficients. Aligar. Bull. Math. I, 25-29. (From pp. 650, 657)

SIDDIQI, A.H. , and GUPTA, S.C.

[1973/74] On the Approximation by Walsh Functions. Aligar. Bull. Math. 3/4, 37-42.

SIMON, P.

[1973] Verallgemeinerten Walsh-Fourier Reihen, I. Annales Univ. Sci. Budapest. Eötvös, Sect. Math. 16, 103-113. (From H. 634)

[1976] VeralTgemeiñerten WaTsh-Fourier Reihen, II. Acta Math. Acad. Sci. Hungar. 27, 329-341. (From p. 636)

[1978a] On a Maximal Function. Annales Univ. Sci. Budapest. Eötvös, Sect. Math. $21,41-44$. (From p. 637)

[1978b] On the Concept of a Conjugate Function. Fourier Analys is and Approximation Theory (Proc. Colloq., Budapest, 1976), Vol. II, pp. 747-755, Collog. Math. Soc. Janos Bolyai, 19, North-Holland, Ams terdam. (From p. 637)

[1979] On the Convergence of Vilenkin-Fourier Series. Acta Math. Acad. Sci. Hungar. 33, 189-196. (From p. 637)

SJÖLIN, P.

[1969] An Inequality of Paley and Convergence a.e. of Walsh-Fourier Series. Arkiv für Math. 7, 551-570. (From p. 631)

SKVORCOV, V.A.

[1968] Haar Series with Convergent Subsequences of Partial Sums. Dokl. HKad. Nauk SSSR, 183 №. 4, 784-786. (From p. 659)

[1973a] Somé Generalizations of Uniqueness Theorems for Walsh Series. Mat. Zametki 13, 367-372. (From p. 660)

[073b] The Coefficients of Multiple Haar and Walsh Series. Vestnik Moskov. Univ. Ser. I Mat. Meh. 28, 77-79. (From p. 647)

[1974] The Uniquenes of WaTsh Series with Convergent Subsequences of Partial Sums. Mat. Zametki 16, 27-32. (From p. 659)

[1975] An Example of a WaTsh Series with a Subsequence of Partial Sums that Converges to Zero Everywhere. Mat. Sbornik (N.S.) 97, 517-539. (From p. 659)

[1976a] An Example of a Zero-series in the Walsh System. Mat. Zametki 19, 179-186. (From p. 661)

[1976b] Uniqueness Theorems for Walsh Series That are Summable by the $(C, 1)$ Method. Vestnik Moskov. Univ. Ser. I Mat. Meh. 31, 73-80. (From p. 660)

[1977a] Uniqueness Condition for the Representation of Functions by Walsh Series. Mat. Zametki 21, 187-197. (From p. 659)

[1977b] The $h$-measure of M-sets for the Walsh Systems. Mat. Zametki 21. 335-340. (From p. 658)

[1977c] The Rate of Convergence to Zero of Coefficients of Zero-series in the Haar and Walsh Systems. Izv. Akad. Nauk SSSR, Ser. Mat. 41, 703-716. (From p. 661)

[1979] Null Series with Respect to a Multiplicative System. Vestnik Moskov. Univ. Ser. I Mat. Meh. №. 6, 63-67. (From p. 661) 
[1980a] Walsh Series That are Convergent with Respect to Partial Sums. Mat. Zametki 28, 45-52. (From p. 659)

[080b] On an Example of a Series in the Walsh System. Mat. Zametki 28, 737748. (From p. 659)

[1981a] Certain Estimates of Approximation of Functions by Cesaro Means of Walsh-Fourier Series. Mat. Zametki 29, 539-547. (From p. 642)

[1981b] On Fourier Series with Respect to the Walsh-Kaczmarz System. Analys is Math. 7, 141-150. (From pp. 636, 639)

[081c] Convergence in $L$ of Fourier Series with Respect to the Walsh-Kaczmarcz System. Vestnik Moskov. Univ. Ser. I Mat. Meh., No. 6, 3-6. (From p. 637)

[1982] Cesaro Means of Fourier Sys tems with Respect to Multiplicative Systems. Vestnik Moskov. Univ. Ser. I Mat. Men., 7-11. (From p. 642)

SKVORCOV, V.A., and WADE, W.R.

[1979] Generalizations of Some Results Concerning Walsh Series and the Dyadic Derivative. Analys is Math. $5,249-255$. (From pp. 655, 656)

SPLETTSTÖSSER, W.

[1979] Error Estimates for Sampling Approximation of Non-Bandlimited Functions. Math. Meth. in the Appl. Sci. 1, 127-137. (From p. 631)

STEIN, E.M. , and WEISS, G.

[1971] Introduction to Fourier Analys is on Euclidean Spaces, Princeton Univ. Press. Prínceton, N.J., p. 53 . (From p. 637)

SAGINJAN, L.A.

[1974] On Indefinite Limits and Sets of Limit Functions of Walsh Series. Mat Sbornik (N.S.) 95, 263-271. (From p. 649)

[1976] Multiple WaTsh-Fourier Series. Soobšc. A.kad. Nauk Gruz. SSR 81, 565567.

[1979] Summability to Infinity of Trigonometric Series of Series in the Walsh System. Mat. Sbornik (N.S.) 108(150), 457-470. (From p. 648)

[1981] On a Theorem of Marcinkiewicz-Zygmund. Izv. Akad. Nauk Armjan. SSR 16 №. I, 54-64. (From p. 649)

ŠARǍ̌ENIDZE, T,V.

[1973] The Strong Summability of Fourier-Walsh Series. Soobšč. Akad. Gruz. SSR 70, 33-36. (From p. 642)

[1976] MuTtipie Walsh-Fourier Series. Soobšc. Akad. Gruz. SSR 81, 565-567. (From p. 642)

ŠNEIDER, A.A.

[1949] Uniqueness of Expansions with Respect to the Walsh System of Functions. Mat. Sbornik 24, 279-300. (From pp. 628, 658)

TAIBLESON, M.H.

[1975] Fourier Analysis on Local Fields, Mathematical Notes, Princeton Univ. Press, Princeton, N.J. (From p. 630)

TAKAHASHI, SH.

[1975] A Statistical Property of Walsh Functions. Studia Sci. Math. Hungar. 10 93-98. (From p. 646)

TALALJAN, A.A.

[1960] The Representation of Measurable Functions by Series. Uspehi Mat. Nauk 15, 77-141. (From p. 648)

[1970] Representation and Uniqueness Problems in the Theory of Orthogonal Series. J. Soviet Math. I (1973), 635-676. 
TALALJAN, A.A., and ARUTUNJAN, F.G.

[1965] Convergence of Series with Respect to the Haar System to to. Mat. Sbornik 66, 240-247. (From p. 648)

TANDORI, $K$.

[1966] Über die Divergenz der Walshschen Reihen. Acta Sci. Math. (Szeged) 27, 261-263. (From p. 634)

TATEOKA, J.

[1978] On the Localization Property of the Double Walsh-Fourier Series. Studia Sci. Math. Hungar. 11(1976), 407-411. (From p. 643)

TEVZADZE, V.I.

[1978] Uniform Convergence of Walsh-Fourier Series. Soobకc. Akad. Nauk Gruz. SSR 92, 301-304. (From p. 640)

[1981] Uniform Convergence of Cesaro Means of Negative Order of Fourier-Walsh Series. Soobšz. Akad. Nauk Gruz. SSR 102, 33-36. (From p. 643)

UL'JANOV, P.L.

[1972] Representations of Functions by Series and Classes $\Phi(L)$. Russian Math Surveys 27, 1-54. (From p. 625)

VILENKIN, N. YA.

[1947] A Class of Complete Orthonormal Systems. Izv. Akad. Nauk SSSR, Ser. Mat. 11, 363-400. (From pp. 629, 639)

VILENKIN, N. YA., and RUBINŠTEIN, A.

[1975] A Theorem of S.B. Stečkin on Absolute Convergence and Character Series for a Zero-Dimensional, Abelian Group. Izv. Vysక. UČeb. Zaved. (160) №. 9, 3-9. (From pp. 638, 651)

WADE, W.R.

[1971] Summing Closed Usets for Walsh Series. Proc. Amer. Math. Soc. 29, 123-125. (From p. 658)

[1975] Uniqueness and $\alpha$-Capacity on the Group $2^{\omega}$. Trans. Amer. Math. Soc. 208, 309-315. (From p. 659)

[1977] Growth Conditions and Uniqueness for Walsh Series. Mich. Math. ‥ 24, 153-155. (From p. 660)

[1979a] Sets of Uniqueness for the Group of Integers of a p-Series Field. Canad. J. Math. 31, 858-866. (From p. 658)

[079b] WaTsh Series and Growth of Functions on Nested Dyadic Intervals. $J$. Indian Math. Soc. 43, 1-11. (From p. 652)

[1980] Uniqueness of Waish Series Which Satisfy an Averaged Growth Condition. SIAM J. Math. Anal. 11, 933-937. (From p. 660)

[1982] [ocalTy Constant Dyadic Derivatives. Periodica Math. Hung. 13, 71-74. (From pp. 647, 656)

[1983] Walsh-Fourier Coefficients and Locally Constant Functions. Proc. Amer. Math. Soc., to appear. (From p. 652)

WADE, W.R., and YONEDA, K.

[1982] Uniqueness and Quasi-measures on the Group of Integers of a p-Series Field. Proc. Amer. Math. Soc. 84, 202-206.

WATARI, $\mathrm{CH}$.

[1958] On Generalized Walsh-Fourier Series. Tôhoku Math. J. 10, 211-241. (From p. 636)

WATERMAN, D.

[1969] W-systems are the Walsh Functions. Bull. Amer. Math. Soc. 75, 139-142. (From p. 626)

[1982] On Systems of Functions Resembling the Walsh System. Michigan Math. 29, 83-87. (From p. 626) 
WEI, YI SU

[1980] On an Extremum Problem for n-Variable Walsh Transforms. Nanjing Daxue Xuebao №. 2 , 6-14.

YANO, SH.

[1951a] On Walsh Series. Tôhoku Math. J. 3, 223-242. (From p. 657)

[1951b] On Approximation by Walsh Functions. Proc. Amer. Math. Suc. 2, 962-967. (From p. 642)

YONEDA, $K$.

[1973] On the Absolute Convergence of Walsh-Fourier Series. Math. Japon. 18, 71-78. (From p. 637)

[1982] Perfect Sets of Uniqueness on the Group $2^{\omega}$. Canad. J. Math. 34, 759-764. (From p. 658)

YOUNG, WO-SANG

[1974a] On Rearrangements of Walsh-Fourier Series and Hardy-Littlewood Type Maximal Inequalities. Bull. Amer. Math. Soc. 80, 490-494. (From p. 635)

[1974b] On a.e. Convergence of Walsh-Kaczmarcz-Fourier Series. Proc. Amer. Math. Soc. 44, 353-358. (From p. 635)

[1976a] Mean Convergence of Generalized Walsh-Fourier Series. Trans. Amer. Math. Soc. 218, 311-320. (From p. 636)

[1976b] A Note on Walsh-Fourier Series. Proc. Amer. Math. Soc. 59, 305-310. (From p. 647)

ZHENG, WEI-XING

[1979] The Generalized Walsh Transform and an Extremum Problem. Acta Math. Sinica 22, 362-374.

ZOTIKOV, S.V.

[1976] On a Class of Systems of Rademacher Type. Izv. Vysš. Ućeb. Zaved. Mat. (170) №. ․․ 30-43. (From p. 644) 


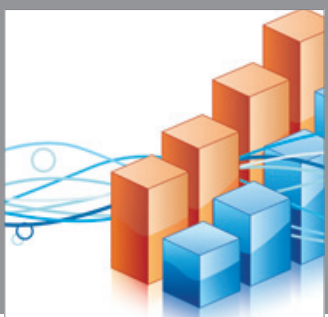

Advances in

Operations Research

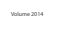

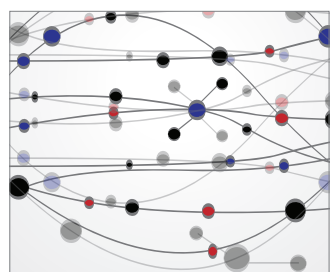

\section{The Scientific} World Journal
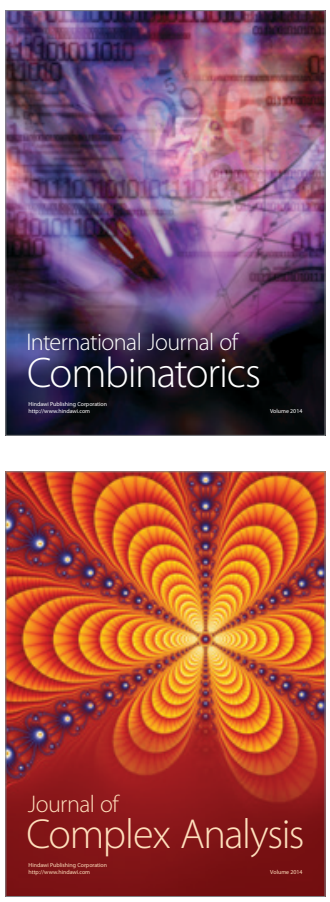

International Journal of

Mathematics and

Mathematical

Sciences
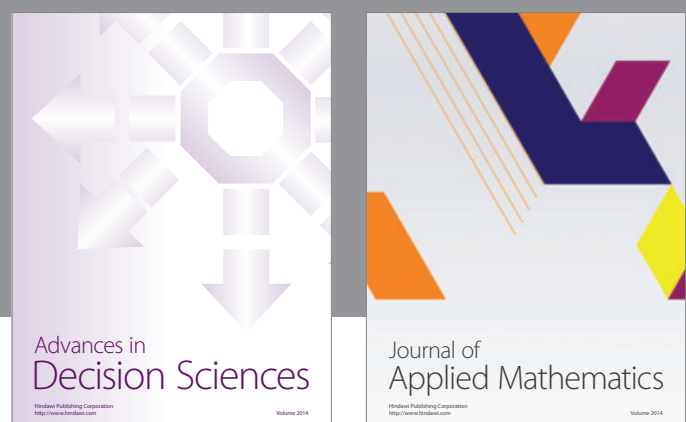

Journal of

Applied Mathematics
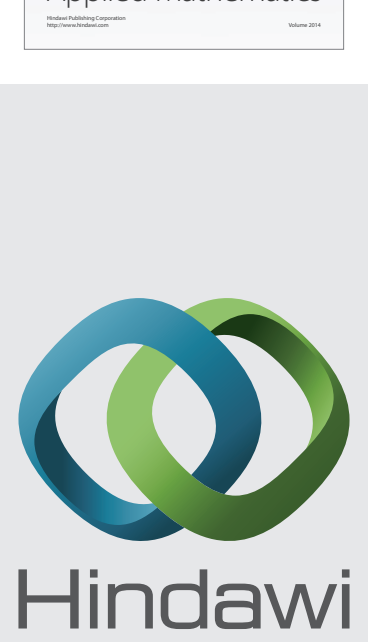

Submit your manuscripts at http://www.hindawi.com
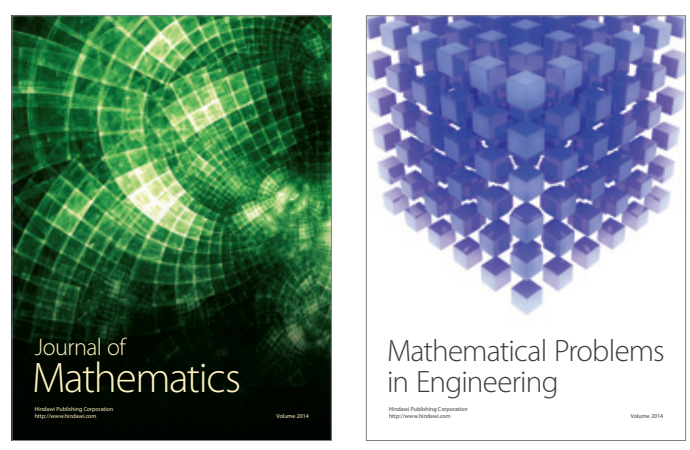

Mathematical Problems in Engineering
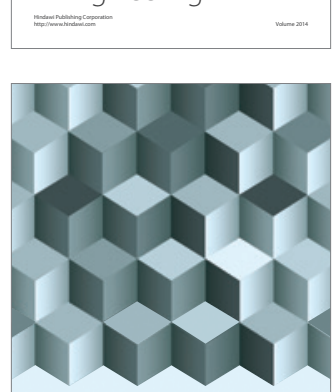

Journal of

Function Spaces
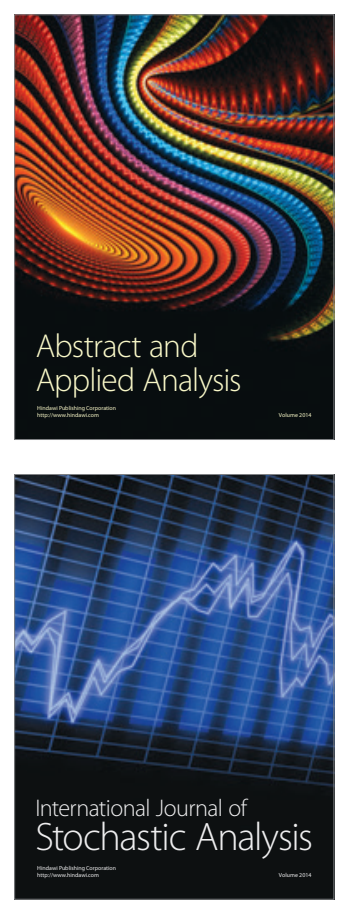

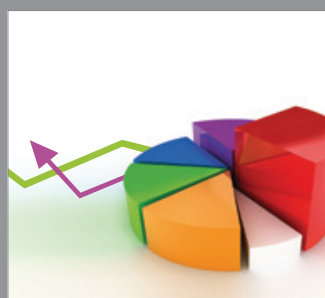

ournal of

Probability and Statistics

Promensencen
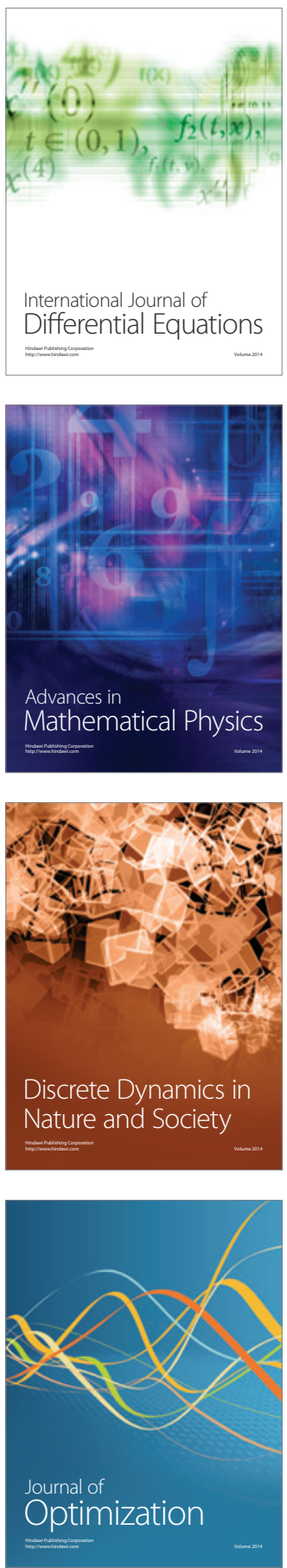